\title{
Die Wirklichkeit der Neonazis
}

Extremistische Kommunikation in der

demokratischen Gesellschaft

\section{Kommunikation unter den Bedingungen ihrer Ablehnung}

Neonazis sprechen und schreiben nicht einfach die Sprache der Nationalsozialisten. Thre Kommunikation ist keine schlichte Neuauflage des historischen Vorbildes. Zwar findet derjenige, der die Flugblatter und Flugschriften, die Pamphlete und Zeitschriften, die Broschüren und die Bücher studiert, immer wieder deutliche Anklänge an die NS-Sprache und bemerkt bekannte Topoi der Argumentation. Zwar entdeckt man, wenn man die entsprechende Medienlandschaft durchstreift, immer wieder ahnliche Muster der Abwertung in Wort und Bild, sieht sich mit Formeln aus dem NSDAP-Parteiprogramm, Material aus dem Propagandaministerium von Joseph Goebbels oder Karikaturen und Schlagzeilen konfrontiert, die aus den Hetzschriften eines Julius Streicher stammen. Das alles gibt es. Neu und anders ist jedoch, dass Neonazis in der Gegenwart unter spezifischen Bedingungen kommunizieren, die ihren sprachlichen und argumentativen Mustern ein besonderes Geprăge geben. Sie praktizieren eine Form der Kommunikation, die wesentlich unter den Bedingungen ihrer offentlichen Ablehnung geschieht: Es besteht, so zeigen Umfragen, Großdemonstrationen und die Stellungnahmen von Medien und Politikern, Gewerkschaften und Kirchen, eine weitgehende gesellschaftliche Isolation neonazistischer und rechtsextremistischer Positionen. ${ }^{1}$

Wer heute NS-Propaganda betreibt, der tut dies nach dem Zivilisationsbruch von Auschwitz. Wer heute den Nationalsozialismus verteidigt oder für eine wie auch immer modifizierte Neuauflage votiert, der tut dies in einer Republik, die sich nicht nur in ihren offentlichen Debatten und Bildungsprogrammen, sondern auch mit den Mitteln der Strafverfolgung und dem Einsatz von Polizei und Verfassungsschutz gegen jede Form der neonazistischen Aktivität zur Wehr setzt. Das Grundgesetz umfasst auch

1 Zu der öffentlichen Wahrnehmung des Rechtsextremismus hält Backes (1998, S. 29) fest: „An der weitgehenden, von meinungsführenden Schichten getragenen gesellschaftlichen Isolation des Rechtsextremismus hat sich seit der deutschen Vereinigung nichts geändert. Dies zeigt beispielsweise das Ergebnis einer Meinungsbefragung aus dem Jahr 1992. Bei der Frage nach Personengruppen, die man nicht gerne als Nachbam haben wolle, setzten die meisten die Gruppe der Rechtsextremisten an die oberste Stelle (77 Prozent), vor Drogenabhängigen (67 Prozent), Trunkslichtigen (66 Prozent) und Linksextremisten (61 Prozent)." 
Artikel zu seinem Schutz wie das Verbot verfassungswidriger Vereinigungen oder Parteien und die Verwirkung von Grundrechten. Die'Bundesprüfstelle für jugendgefährdende Schriften hat die Moglichkeit, Medien auf den Index zu setzen und auf diese Weise den Verkauf von Büchern, Zeitschriften, Videokassetten usw. an Jugendliche zu verbieten. Auch die strafrechtlich relevanten Paragraphen (z.B. Verwenden von Kennzeichen verfassungswidriger Organisationen, Verunglimpfung des Staates und seiner Symbole, Volksverhetzung, Aufstachelung zum Rassenhass) sind vielen mehr oder minder offentlich publizierenden Neonazis vertraut. Das heißt, dass der gesellschaftliche Wirkungszusammenhang, in dem sich Neonazis befinden, eine Reihe charakteristischer Merkmale besitzt, die allesamt in eine ähnliche Richtung zielen: Ablehnung ihrer Meinungen, Verdammnis ihrer Agitation, Entsetzen über ihr offentliches Auftreten und die verbrecherischen Handlungen von Personen und Gruppen, die aus dem Umfeld dieser extremistischen KleinstParteien, der Vereine und Freundeskreise, der illegal operierenden Zellen und der paramilitărischen Zirkel stammen.

Die These dieses Beitrages, die ich in den folgenden Ausfiuhrungen entfalten möchte, lautet, dass dieser Wirkungszusammenhang relevant ist, wenn man die Sprach- und Argumentationspraxis der Neonazis überhaupt verstehen und seine eigentümliche Fremdbestimmtheit erklaren will. ${ }^{2}$ Die neonazistische Kommunikation ist, wie sich zeigen lässt, zu wesentlichen Anteilen die gereizte und hochgradig aggressive Reaktion auf den gesellschaftlichen Umgang mit dem Nationalsozialismus und dem Neonazismus. Man antwortet in ideologischer Weise auf den offentlichen Diskurs ${ }^{3}$ über die NS-Zeit, entwirft eine Gegensprache, versucht eine Diskurskorrektur und betreibt eine Vergangenheitsbewältigung der ganz eigenen Art. Wenn man die Muster und Verflechtungen zwischen den neonazistischen Aktivitäten, der offentlichen Reaktion und dem staatlichem Kontrollhandeln beobachtet und analysiert, dann zeigt sich, dass die Kommunikation der Neonazis förmlich an die Wertungen ihrer Gegner gefesselt ist: Sie folgt einem umfassenden Programm der

2 Eine deutlich kürzere Fassung dieses Beitrags, dessen wesentliche Ergebnisse auf meine Dissertation zurückgehen, ist auch in der Festschrift für Jörg Hennig publiziert worden: Siehe insgesamt Pörksen, B. (2000) und Pörksen, B. (2001), S. 311-337.

3 Der Begriff des Diskurses bezieht sich ausdrücklich nicht auf Michel Focaults anthropologisch hochgradig voraussetzungsvolle Arbeiten (Stichwort: Leugnung des autonomen Subjekts) und die in Gefolge seiner Studien entstandenen Mischformen aus politischer Überzeugungsarbeit und wissenschaftlicher Sprachanalyse, die wichtige Themen innerhalb der Sprachwissenschaft eher zu marginalisieren drohen. „Diskurs' meint hier schlicht: Redeweise über ein Thema. 
Exkulpation und dient der Abwehr von Vorwürfen, die von einer demokratisch gesinnten Öffentlichkeit vorgegeben werden.

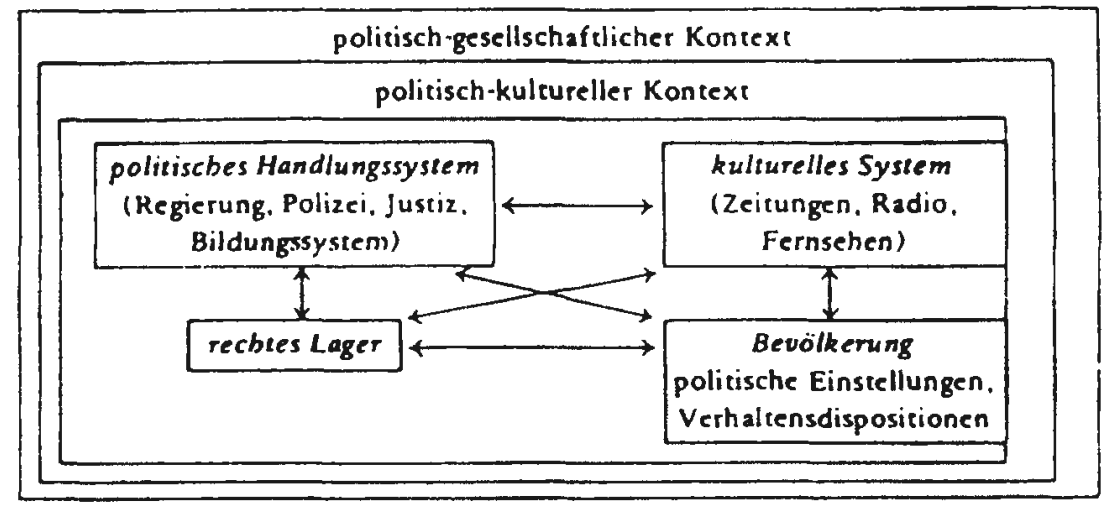

Die Abblldung zeigt eine schematische Darstellung des Wirkungszusammenhangs, in dem sich der organisierte Rechtsextremismus nach 1945 befindet. Zentral ist die Interdependenz von rechtsextremer Politik, öffentlicher Reaktion und staatlichem Kontrallhandeln. (Entnommen aus: Dudek/Jaschke 1984, S. 30).

\section{Medien und Materialbasis}

Die Sprachprodukte der Neonazis erreichen jene, die sie erreichen sollen, und jene, die sich für sie interessieren, auf sehr verschiedenen Wegen. Neonazistische Parolen tauchen seit dem Ende der 80er Jahre in Computerspielen auf; im so genannten Thule-Netz, einem bundesweiten Verbund von Hobbymailboxen, finden sich genauso wie im Internet programmatische Schriften. Anrufbeantworter mit entsprechenden Ansagen, $\mathrm{Na}$ tionale Infotelefone genannt, enthalten Kommentierungen des Tagesgeschehens, Kontaktadressen und Telefonnummern von Gesinnungsgenossen. Und schließlich gibt es, neben der auf Versammlungen und Gruppentreffen stattfindenden mündlichen Kommunikation, zahlreiche gedruckte Medien, Zeitungen und Zeitschriften, Programme, Mitteilungen, Broschüren und Bücher. Sie unterscheiden sich nach der Höhe der Auflage, dem Grad der formalen und gestalterischen Professionalitat, den thematisch bevorzugten Schwerpunkten und dem Legalitătsstatus. Die Autoren, die in den neonazistischen Medien des Printbereichs veroffentlichen, zeichnen sich jedoch allesamt durch ein ihnen gemeinsames Set von Überzeugungen aus, das ihre Wirklichkeitskonstruktion steuert. Sie beziehen sich ohne erkennbare Distanz positiv auf zentrale Ideologeme des Nationalsozialismus, die sie reaktiv gegen offentliche Einschătzungen verteidigen. Sie propagieren ausländerfeindliche Thesen, vertreten einen rassistisch unterlegten Volksbegriff, einen rassistischen Antisemi- 
tismus, ein sozialdarwinistisch geprägtes Geschichtsbild und bedienen sich verschwörungstheoretischer Konzeptionen. Der Wahrheitsanspruch, der in ihren Texten offenbar wird, ist hăufig absoluter Natur; man formuliert keine Deutungsangebote, sondern Gewissheiten mit dem Anspruch der Letztgültigkeit. Gewalt als Mittel der Politik und der Auseinandersetzung wird keineswegs geächtet, sondern befürwortet. Man diffamiert in neonazistischen Medien die Repräsentanten der bundesdeutschen Demokratie und lehnt wesentliche Grundprinzipien der westlichen Demokratien (Pressefreiheit, Gleichheit der Menschen etc.) ab. ${ }^{4}$

Die Sprach- und Argumentationsmuster, die sich in diesem Feld der neonazistischen Publizistik beobachten lassen und die hier analysiert werden sollen, entstammen einem bewusst gewählten Zeitraum: Er umfasst die Jahre von 1989 bis 1993. Diese Phase ist, gerade wenn es um die kommunikativen Interaktionen zwischen den Neonazis und der sie umgebenden Gesellschaft geht, besonders aufschlussreich, da es in diesen vier Jahren zu einer bis dahin unbekannten Präsenz von Neonazis in der Öffentlichkeit kam: Neonazis agitierten in den neuen Bundesländern, besetzten Häuser und veranstalteten Kongresse und Aufmärsche und riefen dazu auf, die Wohnheime von Asylbewerbern anzugreifen. Gleichzeitig explodierte die Zahl der neonazistisch und rechtsextremistisch motivierten Gewalttaten. Die Öffentlichkeit reagierte und setzte ein Zeichen mit Demonstrationen und Lichterketten. Es folgten Prozesse, Veranstaltungs- und Parteienverbote; zahlreiche Anführer und Mitglieder neonazistischer Gruppen mussten vor Gericht. Und es sind diese Reaktionen und die Maßnahmen einer um ihr Ansehen und ihre Zivilităt besorgten Gesellschaft, die sich ihrerseits in den neonazistischen Schriften spiegeln: Sie bedingen (im Verbund mit dem allgemeinen gesellschaftlichen Diskurs über den Nationalsozialismus) einen besonderen Gebrauch der Ideologiesprache. ${ }^{5}$ Sie erzeugen (zusammen mit dem Versuch der univer-

Bei der neonazistischen Spielform des Rechtsextremismus handelt es sich nicht um eine systematisch ausgearbeitete Ideologie, sondern um eine totalitäre Weltanschauung mit Konglomeratcharakter, die gleichwohl deutlich von anderen rechtsextremen Gesinnungen abgrenzbar ist. Diejenigen, die sie vertreten, gehören einer gemeinsamen ideologischen Gruppe an; sie haben ein System der Wirklichkeitsinterpretation gemeinsam. Siehe Pörksen, B. (2000), S. 41-42 und S. $67 \mathrm{ff}$. Ideologiesprachen gehören zu einem gruppensprachlichen Prototyp, den ich - im Unterschied zu Fach-, Sonder- und Berufssprachen - ,Weltanschauungssprache* nennen möchte: Sie haben die Funktion der gruppeninternen Stabilisierung und der gruppenexternen Beeinflussung, der Mitgliederkonsolidierung und der Mitgliederwerbung. Sie werden von ideologischen und politischen Gruppen, Sekten und missionsbereiten Glaubensgemeinschaften gesprochen und geschrieben und müssen in Relation zu den zugrundeliegenden Glaubens- und Deutungssystemen interpretiert werden. 
salen Schuldabwehr) einen spezifischen Einsatz der jeweiligen Argumentationsfiguren.

\section{Arbeit am Image: Versuche der Exkulpation}

Die neonazistische Ideologie ist, wie dies die nachfolgende Analyse zeigen wird, in einem hohen Maße reaktiv. Man reagiert gereizt auf die öffentliche Meinung, benutzt einen Großteil der agitatorischen Energie, um den Nationalsozialismus und den Neonazismus vom Makel des Verbrecherischen und dem Stigma des Unmoralischen zu befreien und setzt sich exzessiv mit der Wahrnehmung der eigenen Gesinnung in den Medien auseinander. Das ist verständlich. Ein volliger Rückzug in die Welt der eigenen Ideologie und ein Verlust des Außenkontakts würde auch dem Ziel der Persuasion widersprechen, das die politisch-ideologische Kommunikation insgesamt auszeichnet (Grünert 1983, S. 44). Der Grund für die Auseinandersetzung mit dem eigenen Image leuchtet schon deshalb unmittelbar ein: Jeder, der wirken will, muss sich zuerst darüber klar werden, wie er wirkt, welche Auffassungen über ihn und seine Sicht der Dinge bereits verbreitet sind. Auf die öffentlichen Bilder und Deutungen, die Urteile und Verurteilungen versuchen Neonazis zu reagieren, um massiv auf sie einzuwirken und sie zu korrigieren. Sie benutzen zu diesem Zweck eine Reihe von gedanklichen, argumentativen und sprachlichen Mustern: Diese haben nicht notwendig den Charakter ausgefalteter Argumentationen mit explizit formulierten Behauptungen und entsprechend klar gekennzeichneten Begründungen, sondern sie sind oft weniger deutlich in ihrem Charakter als Überzeugungsversuche erkennbar: Sie enthalten zahlreiche Prämissen und persuasive Wirkungspotentiale, die es erst aufzudecken gilt. ${ }^{6}$ Sie enthalten ein gruppenspezifisches Ideologiewissen, das man beschreiben muss, um das Gesagte und Gemeinte auch außerhalb der Gruppe verständlich zu machen. Um eine Fülle persuasiver Phänomene in den Blick zu bekommen, ist in den folgenden Abschnitten der Analyse etwas unscharf von, gedanklichen und argumentativen Mustern' und von ,sprachlichen und argumentativen Mus-

6 Um nicht in die Gefahr zu geraten, die Macht der Sprache zu über- und die Mlindigkeit der Emittenten und Rezipienten zu unterschätzen, sollen hier einzelne Äußerungen und ganze Texte als ,Wirkungspotentiale' aufge fasst werden. Sämtliche Aussagen und Interpretationen beziehen sich somit auf die Möglichkeit, die Eventualität einer entsprechenden Wirkung. Was die zu analysierenden Beispiele tatsächlich auslösen, wen sie verführen, manipulieren, zum Hass und zur Gewalt aufstacheln, kann hier nicht geklärt werden. Zum Begriff des Wirkungspotentials siehe Hennig (1994), S. 1. 
tern' die Rede. Sie konstituieren sich durch gruppenintern verbindliche, immer wiederkehrende Formen des Schreibens und Redens. Und man muss sie zu der offentlichen Diskussion über den Nationalsozialismus und den Neonazismus in Beziehung setzen: Offenbar wird dann, wenn man diesem doppelten Blick auf die extremistische Subkultur und die demokratische Mehrheit folgt, eine Imagearbeit in neonazistischen Publikationen, die auf restlose Exkulpation und auf die Umkehrung sämtlicher Vorwürfe zielt.

\subsection{Gedankliche und argumentative Muster}

\section{Verschwörungstheorien: Weltformeln des Übels}

Verschworrungstheorien werden von Neonazis u.a. zur Schuldabwehr und einer fundamentalen Apologie des Nationalsozialismus eingesetzt. So behauptet man etwa, der Holocaust habe gar nicht in der von überlebenden Augenzeugen, den Tătern selbst und zahllosen Historikern beschriebenen Weise stattgefunden: Man spricht von einer "Gaskammerlüge "7 und verbreitet die inzwischen auch durch das Strafrecht erfasste Behauptung, die Gaskammern seien von jüdischen Weltverschwörern erfunden worden, um Zahlungen zu erpressen. ${ }^{8}$ Hermann Bott hat für derartige Behauptungen, die eine lange Tradition besitzen, den Begriff ,Entlastungs-Antisemitismus' vorgeschlagen (Bott 1969, S. 108-111). Er ist geschickt gewählt und trifft die Sache, weil er auf die zwei wesentlichen Züge dieser Spielform der Apologetik durch Leugnung verweist. Zum einen geht es darum, von den nationalsozialistischen Verbrechen zu entlasten, um den verwandten Ideologemen des neonazistischen Rechtsextremismus wieder zu erneuter Akzeptanz zu verhelfen. Zum anderen ist diese Entlastung gleichzeitig mit einer neuen Spielform des Antisemitismus verbunden, der letztlich den Juden selbst ihre Vernichtung in den Konzentrationslagern zum Vorwurf macht und auf eine schlichte Verkeh-

7 F. 25, S. 1, 2. 34. Randbemerkung zur Zitierweise: Zitate aus verschiedenen undatierten und anonym veröffentlichten Flugblättern und Flugschriften, die auf den neonazistischen Freundeskreises Frethelt für Deutschland zurückgehen, weise ich nach, indem jeweils - wie in dem vorstehend genannten Beispiel - die Flugblatt- oder Flugschriftennummer genannt wird, dann die Seiten- und schließlich die Zeilenzahl. Die auf diese Weise zitierten Schriften können in der Bibliothek des Germanischen Seminars der Hamburger Universität in Form eines Materialbandes eingesehen werden. Andere Organe werden zitiert, indem jeweils der Name des Organs und falls vorhanden - der Name des Autors, die Heftnummer und das abgekürzte Jahr der Veröffentlichung genannt werden. 2usätzliche Angaben finden sich im Literaturverzeichnis; wenn der Autor nicht genannt ist, wird dieser im Literaturverzeichnis als Anonym etikettiert.

8 Siehe insgesamt F. 45, S. 1-4. 
rung des Tăter-0pfer-Verhăltnisses hinausläuft. Nicht der Mörder, sondern die Gruppe, zu der der Ermordete gehort hat, wird schuldig gespro. chen. Die Schreckensbilder des Vollkermordes reduzieren sich auf eine zur Geschichtsverdrängung eingesetzte Vorstellung von einer jüdischen Weltverschwörung. Eine derartige gedankliche Konstruktion hat aus der Sicht der neonazistischen Gruppe den Vorteil, dass sie nicht widerlegt werden kann. Sie ist, um einen Begriff Karl Poppers aufzugreifen, ,selbstimmunisierend' (siehe Pörksen, B., 1997, S. 394). Verschwörungstheorien scheinen gegen rationale Widerlegungsanstrengungen immun zu sein, da auch ihre Nichtbeweisbarkeit zum scheinbaren Beweis für die geheimnisvolle Perfidie der Verschwörer, die raffiniert alle Spuren verwischen, umgedeutet werden kann. Eine Verschworung ist eben naturgemäß ein Mysterium, das sich der rationalen Durchdringung entzieht. Thre Wirkungsmacht bezieht sie "aus dem Geheimnis, das sie umgibt" (Piper 1995, S. 127). Noch so heterogene Ereignisse können unter der Prämisse einer weltweiten Verschwörung in eine scheinbar ursächliche Verbindung gebracht werden; auch die Verbrechen des NS-Regimes lassen sich auf diese Weise relativieren. Und so ist es kaum erstaunlich, dass Neonazis bei ihren Versuchen, das NS-Regime moralisch zu exkulpieren, immer wieder auf Konspirationstheorien zurückgreifen. Sie sind vielseitig verwendbar, selbstimmunisierend und dispensieren von rationaler Argumentation, eignen sich zur Konstruktion kausaler Zusammenhänge und taugen als eine Art Weltformel des Übels: Man kann mit ihrer Hilfe Unangenehmes scheinbar wegerklären.

\section{Schuldabwehr durch Aufrechnung: Unrecht minimiert Unrecht}

Man kann sich fragen, was Neonazis eigentlich zu dem Versuch motiviert, die NS-Verbrechen entweder ganz zu leugnen oder aber sie zu relativieren. Die Antwort ist einfach: Eine Relativierung nationalsozialistischer Unmoral dient der Rettung der entsprechenden Ideologeme als einer heute noch brauchbaren Gegenwartseinstellung. Zu diesem Zweck greift man in neonazistischen Publikationen hăufig auf das argumentative Muster der Aufrechnung zurück, das auf der Prämisse basiert, dass sich ein Unrecht durch anderes Unrecht minimieren lăsst. Ein immer wiederkehrendes Agitationsthema, mit dem sich nicht nur Neonazis, sondern auch zahlreiche andere Rechtsextremisten befassen, ist in diesem Zusammenhang die Zerstörung Dresdens durch Verbände der britischen, der amerikanischen und der kanadischen Luftwaffe: Sie wird in einer Flugschrift als "Holocaust aus der Luft"9 bezeichnet. Man agitiert mit Zahlen, die eine seriöse Geschichtswissenschaft längst widerlegt hat,

9 F. 15, S. 2, 2. 6. 
geht von bis $\mathrm{zu} 500.000$ Opfern der Bombardierungen aus ${ }^{10}$ - nicht rechtsextreme Historiker sprechen von 35.000 Toten (Mayr 1993, S. 61f.) - und greift insgesamt bevorzugt auf die Arbeiten von David Irving zurïck, der in neonazistischen Gruppierungen als wissenschaftliche Koryphäe gilt. Auch er leugnet inzwischen den Holocaust. Durch den Hinweis auf eine fremde Schuld und eine Aufrechnung, die die unterschiedlichsten Untaten in der Geschichte der Menschheit mit den Verbrechen der Nationalsozialisten auf eine Stufe stellt, soll der Nationalsozialismus exkulpiert werden. Man verweist wahlweise auch auf das Schicksal der nordamerikanischen Indianer, den Abwurf der Atombombe, den Krieg in Vietnam oder eben immer wieder auf die Zerstorung Dresdens. Der Gedankengang, der sich bei den neonazistischen Autoren beobachten lässt, ist zu Ende, wenn man das gewünschte Ergebnis der Schuldminimierung erreicht zu haben glaubt. Das Bemühen um den Vergleich wird dann abgebrochen, denn es geht nicht darum, in einem Akt der Gegenüberstellung, Unterschiede herauszuarbeiten, sondern Vergehen und Verbrechen unterschiedslos gleichzusetzen, um die Inkriminierung der eigenen Gesinnungsgenossen zu beenden.

\section{Anpassung an abgelehnte Werte: die Entwurzelungsthese}

Im September des Jahre 1973 schrieb der ehemalige persönliche Pressereferent von Joseph Goebbels, Wilfried von Oven, in der Zeitschrift LaPlata-Ruf, man solle die eigenen Aussagen so gestalten, dass "sie nicht mehr ins Klischee des ,Ewig-Gestrigen' passen“ (zit. nach Dietzsch, 1988, S. 33). Es heißt in dieser Zeitschrift:

"In der Fremdarbeiter-Frage etwa erntet man mit der Argumentation ,Die sollen doch heimgehen' nur verständnisloses Grinsen. Aber welcher Linke würde nicht zustimmen, wenn man fordert: „Dem Großkapital muss verboten werden, nur um des Profits willen ganze Völkerscharen in Europa zu verschieben. Der Mensch soll nicht zur Arbeit, sondern die Arbeit zu den Menschen gebracht werden.' Der Sinn bleibt der gleiche: Fremdarbeiter raus! Die Reaktion der Zuhörer wird aber grundverschieden sein." (zit. nach Dietzsch, 1988, S. 34)

Entsprechend und vermutlich als ein spätes Echo auf die hier gegebene Einweisung in das subversive Argumentieren kritisiert man noch im Jahre 1991 in einer neonazistischen Zeitschrift die „Entwurzelung ganzer Bevölkerungskreise, ihre Verschiebung in eine völlig fremde Welt und ihren Missbrauch als Sklaven des Großkapitals". ${ }^{11}$ Ohne eine derartige antikapitalistische Diktion kommen dagegen andere, gleichwohl

10 Siehe insgesamt F. 15.

11 Unabhängige Nachrichten (UN) 7/91, S. 7. 
ähnlich gelagerte Versuche der Persuasion aus, die sich scheinbar am Wertesystem des gegnerischen Lagers orientieren, es bei genauerer Betrachtung jedoch fundamental negieren. So fordert man immer wieder, Ausländer dürften ihrer Heimat nicht entrissen, sie dürften nicht entwurzelt werden. Man beklagt, dass sie ihr Land verlassen mussten, dass sie "ihre Tradition, Kultur, Religion und die eigene staatliche Ordnung“12 verloren haben. Das bedeutet: Man stützt sich erkennbar auf das gruppenextern gültige Normgebilde des ausländerfreundlichen Engagements, um faktisch die Abschiebung und Rückführung der Einwandernden zu propagieren. Sie werden - scheinbar zu ihrem eigenen Nutzen - nach Hause und zurïck in die Heimat geschickt.

\section{Imitation von Wissenschaft: Simulation von Neutralität}

Zur Leugnung des Holocaust kursieren in der neonazistischen Presselandschaft eine Reihe von wissenschaftlich aufgemachten Publikationen. Besonders bekannt geworden ist der so genannte Leuchter-Report, in dem von dem Amerikaner Fred Leuchter (1988) - auf der Basis chemikalischer Untersuchungen einiger Gaskammer-Ruinen in Auschwitz - behauptet wird, die massenhaften Vergasungen seien schon rein technisch unmöglich gewesen. In diesem und in verschiedenen vergleichbaren Gutachten, die man zum Zweck der Exkulpation in Auftrag gegeben hat, wird das wissenschaftliche Vorgehen (das Bemühen um Exaktheit, die Nennung von Belegen und Quellen) rein konnotativ benützt: Das Prådikat wissenschaftlich fungiert hier nicht mehr als Indikator einer um Verifikation oder Falsifikation bemühten Erkenntnismethode, sondern funktioniert allein als ein Prestigesignal. Als der Maßstab, den die fortwährend um die Entlarvung von offentlichen Fehlurteilen bemühten Neonazis ins Spiel bringen, gilt ihnen die geschichtliche Wahrheit. ${ }^{13}$ Das ist, so die Selbsteinschătzung, das Kriterium der eigenen Geschichtsdarstellung. ${ }^{14}$ Wahrheit ist Gruppenbesitz; außerhalb der Grenzen der eigenen Gruppe beginnt die Konfrontation mit den Lügen oder auch der Lüge. ${ }^{15}$ Man

12 Standarte 4/93, S. 6.

13 Siehe exemplarisch F. 26, S. 1, Z. 41.

$14 \mathrm{Ob}$ einzelne Neonazis allerdings tatsächlich von der Leugnung des Holocaust, die sie betreiben, überzeugt sind, ist eine schwierige Frage. So hat Schmidt ein Interview mit Christophersen, dem Verfasser der Broschüre „Die Auschwitz-Lüge“ aufgezeichnet, das deutlich macht, dass dieser wohl nicht an seine eigenen Behauptungen glaubt. Schmidt (1993), S. 341-343.

15 Gelegentlich wird auch, wohl um einer Strafverfolgung auszuweichen, das Wort Lüge im Verbund mit dem bestimmten Artikel als ein ideologiesprachliches Synonym fur Auschwitz-bzw. Gaskammerlüge propagiert. Man schwankt erkennbar zwischen dem Wunsch, semantisch eindeutig zu sein, aber doch juristisch unverfänglich $z \mathfrak{u}$ formulieren. 
möchte dagegen der „Auschwitz-Wahrheit"16 zum Durchbruch verhelfen, beruft sich auf die Augenzeugenberichte von Gesinnungsgenossen und eben immer wieder auf den amerikanischen Gaskammerkonstrukteur Fred Leuchter, den man stets als einen Experten einstuft und präsentiert, obwohl ihm sämtliche Merkmale des Expertenstatus fehlen: Er besitzt nicht die angegebene berufliche Qualifikation und keine historischen Fachkenntnisse und ist finanziell von seinen Auftraggebern abhängig. Gleichwohl wirbt man mit seinen pseudowissenschaftlich aufgemachten Gutachten um Glaubwürdigkeit, benutzt die akademischen Titel einzelner zur Autorisierung des Gesagten, imitiert Textsorten, die durch die formale Gestaltung die Exaktheit der Darlegungen untermauern sollen. Man bezieht sich auf die - vermeintlichen - Aussagen renommierter und anerkannter Institutionen (UNO, Rotes Kreuz) und bildet in der eigenen Medienlandschaft regelrechte Zitier- und Bestătigungskartelle aus, die Realitătsgewissheit durch beständige Wiederholung erzeugen und durch fortwăhrendes wechselseitiges Zitieren eine Quellenvielfalt und enorme Quantität der Belege für die eigenen Behauptungen nahelegen. Berichte von Überlebenden und Zeugen, Fotos und Filme, schriftliche historische Dokumente der Nationalsozialisten und Publikationen anerkannter Historiker werden, wenn sie den eigenen Vorannahmen widersprechen, nicht ernst genommen, gar nicht erst diskutiert oder schlicht als Fälschungen abgetan - und sehr ernst genommen, wenn sie in irgendeinem Detail, die eigenen Vorannahmen zu stützen scheinen. Die Geständnisse und teilweise detaillierten Berichte der Täter über den organisierten Massenmord verwirft man pauschal oder behauptet, sie seien durch Folterungen erpresst worden (zu den Methoden der Holocaustleugner siehe insgesamt Virchow 1996).

\section{Der Appeal des Anerkannten: Textsortenverschränkung}

Gewicht versuchen neonazistische Autoren ihren Annahmen auch dadurch zu verleihen, indem sie auf gesellschaftlich renommierte Zeitungen und Zeitschriften als Quellen zurïckgreifen: Sie zitieren diese ausgiebig und drucken Artikel - oft als Auszug - nach. Die Zeitungen, die man in dieser Weise verwendet, sind in der Regel über den Verdacht, sie reproduzierten rechtsextremistische oder gar neonazistische Ansichten, erhaben. Gerade deshalb eignen sie sich als Autoritäten, da ihre Standpunkte und Deutungen, die man zur Stützung der eigenen hochgradig umstrittenen Position benütat, auch auf eine gruppenexterne Akzeptanz hoffen dürfen. Ein extremer Fall einer solchen Autorisierung durch die Anleihe bei den etablierten Medien kommt in dem nachfolgend abgedruckten

16 Remer-Depesche 3/93, S. 1. 
Flugblatt zum Ausdruck: Hier wird mit zahlreichen Authentizitătssignalen - den Insignien des Originals - gearbeitet, um erneut die Ermordung von Menschen in den Gaskammern des Dritten Reiches abzustreiten.

\section{NENN'S GAS AUSGEHT
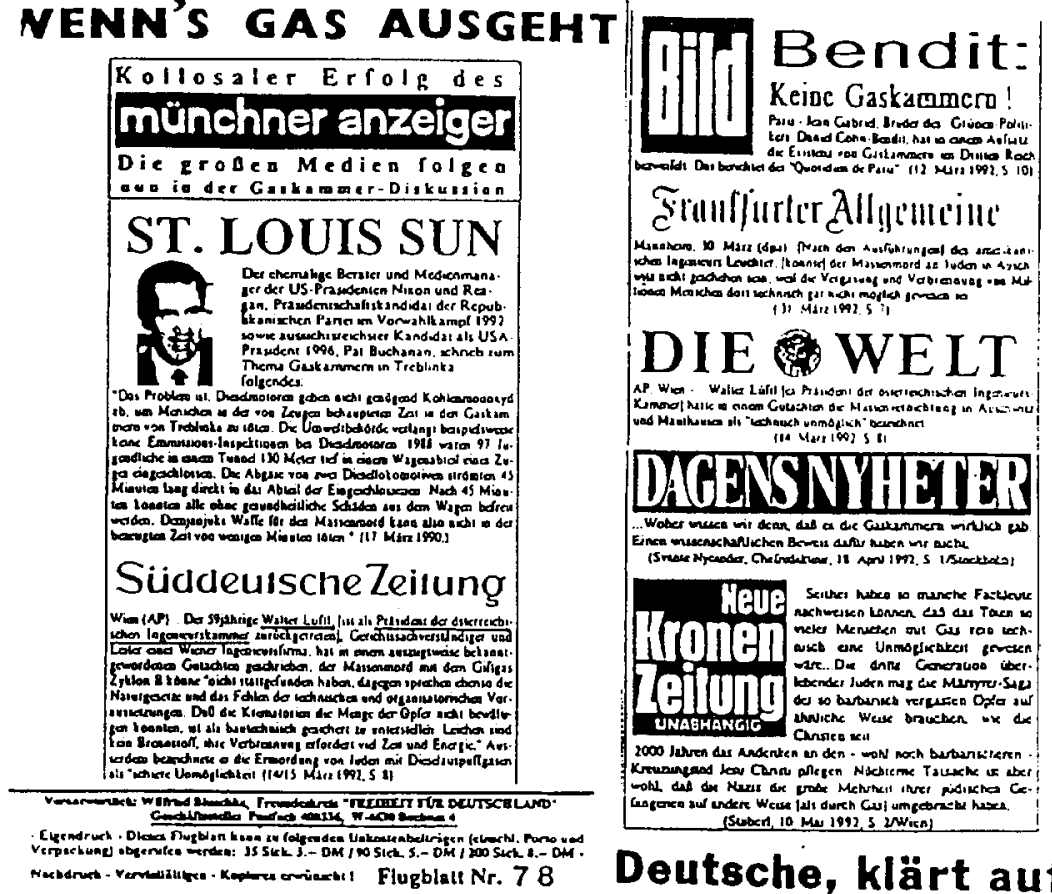 \\ Deutsche, klärt auf!}

Die Abbildung illustriert das Prinzip der Textsortenverschränkung. ${ }^{17}$

Der Text des oben abgebildeten Aufrufs besteht lediglich aus einigen wenigen von der Gruppe selbst verfassten Sätzen (man beachte die Überschrift „Wenn's Gas ausgeht") und verschiedenen Ausschnitten aus Meldungen, Nachrichten und Kommentaren, die mit der imperativischen Formel „Deutsche, klärt auf!“ unterschrieben sind. Aufgrund der Textsortenverschränkung tritt das dominante Ziel der anderen Textsorten (Meldung und Nachricht dienen der Information) in den Hintergrund; diese werden im Gesamtzusammenhang zu Rechtfertigungssequenzen des Aufrufs. Es lässt sich ein Phänomen beobachten, das mit dem Begriff ,Textsortenverschränkung ${ }^{18}$ erfasst werden soll. Textsortenverschrän-

17 Die Vorlage hat in der mir vorliegenden Form leider eine schlechte Qualität.

18 Zur ursprünglichen Verwendung des Begriffs 'Textsortenverschränkung' siehe insgesamt Pörksen, U. (1974). U. Pörksen verwendet den Begriff zur Beschreibung von Spracheffekten als Ergebnis von Normverletzungen. 
kung bezeichnet hier die Kombination und Verschränkung mehrerer Textsorten, wobei dann aus dem Gesamtzusammenhang des so entstandenen (neuen) Textes sein jeweils dominantes Ziel zu erschließen ist, sich also eine Hierarchie der Ziele herausbildet, die Interpretation verlangt, aber gleichwohl einer allgemeinen Gesetzmäßigkeit unterliegt. Es entsteht durch die Textsortenverschränkung eine Sequenz von Sprachhandlungen, deren Bestandteile (= einzelne Textsorten) hierarchisch miteinander verknüpft sind. Der Zweck der untergeordneten, subsidiären Handlung ist es dabei, das mit der übergeordneten, dominierenden Sprachhandlung angestrebte Ziel zu unterstützen. Dieses Phänomen taucht in neonazistischen Medien in verschiedenen Variationen auf: So werden die verschiedensten Textsorten miteinander verschränkt, wobei jeweils die (oftmals etwas gekürzten) Meldungen, Nachrichten und Kommentare aus renommierten Zeitungen faktisch den Status einer autorisierenden oder rechtfertigenden Sequenz einnehmen.

\section{Täterentlastung durch Mitglieder der Opfergruppe: besondere Zeugen}

Neonazis sind antisemitisch, sie erleben aber offenkundig den Vorwurf des Antisemitismus als eine Behinderung ihrer agitatorischen Aktivitäten, als einen Bremsblock der ideologischen Entfaltung. Um dem Vorwurf des Antisemitismus - im Sinne einer präventiven Einwandsbehandlung - auszuweichen und doch weiterhin antisemitische Auffassungen verbreiten zu können, wenden sie deshalb eine besondere Form des Gegnerzitats an: Der Zitierte gehört nicht zur eigenen Gruppe, sondern er erscheint auf den ersten Blick als ideologisch in besonderer Weise unverdächtig, denn er gehört zur Gruppe der Opfer selbst. Er ist - darauf wird dann stets unmissverständlich hingewiesen - jüdischen Glaubens bzw. (nach Auffassung der neonazistischen Autoren) jüdischer Volkszugehörigkeit. So schreibt ein bekannterer Neonazi, der zum Zeitpunkt der Veroffentlichung dieses Artikels einem Gerichtstermin wegen revisionistischer Bestrebungen ins Auge sieht, er würde im Gericht und bei seiner „Beweisführung" von "Josef Burg, welcher jüdischer Abstammung ist" unterstützt; dieser Josef Burg habe „für eine jüdische Organisation kurz nach dem Kriege viele deutsche Konzentrationslager" besucht. Er könne daher „als unmittelbarer Zeitzeuge bedeutende Aussagen machen. "19 Für die in diesem Fall intendierte Leugnung des Holocaust und die offensive Umkehrung des Tăter-0pfer-Verhältnisses wird hier also ein Zeuge präsentiert, der - warum auch immer - bereit ist, die eigene Gruppe anzuklagen. Auch ein anderes Organ, Recht und Wahrheit, zitiert den scheinbar neutralen und daher besonders wichtigen Beistand, der allerdings in neo-

19 Ochensberger, in: Sieg 2/89, S. 2. 
nazistischen Kreisen seit langem als ein notorischer Antisemit für eine gewisse Aufmerksamkeit sorgt. Man nennt ihn hier den „bekannten jüdischen Schriftsteller und Publizisten J. G. Burg". Diesmal vertritt er die verschworrungstheoretisch begründete Behauptung, die Nürnberger Rassengesetze seien „auf Wunsch und Drängen zionistischer Führer erlassen worden. “20

\section{Die Autorität des Gesetzes: die Technik der Legalisienung}

Der Vorwurf der Verfassungsfeindlichkeit, der sich aus der 0pposition zu wesentlichen Gehalten des Grundgesetzes und der demokratischen Ordnung insgesamt - Parteienpluralismus, Gleichheitspostulat, Pressefreiheit etc. - ergibt, ist neonazistischen Autoren wohl vertraut. Man kann hier nur spekulieren, aber vielleicht handelt es sich bei der häufigen Bezugnahme auf das Grundgesetz und die Verfassung ebenfalls um einen Versuch der reaktiven Vorwurfsdemontage: Immer wieder wird - durch aus dem Kontext gerissene Zitate, Umdeutungen und gewichtig klingende Hinweise auf Paragraphen und Eidesformeln - vermeintlich nachgewiesen, dass es sich gerade bei den neonazistischen Aktivitäten und Forderungen um grundgesetzlich abgedeckte Verhaltensweisen und Zielsetzungen handele. Immer wieder taucht etwa, wenn es um die vermeintliche Vorzugsbehandlung der Asylbetrüger und die Forderung oder schlichte Akzeptanz der Einwanderung geht, die Annahme auf, deutsche Politiker würden sich in einem juristischen Sinn schuldig machen. Politische Parteien, die nichts gegen Einwanderer und Asylbetrüger tun, werden als „Verrăter des Grundgesetzes“ eingestuft, da dieses, wie es heißt, „alle Politiker feierlich verpflichtet, ,Schaden vom deutschen Volke abzuwenden'“.21 Die Bundesregierung fördere und bevorzuge Asylbetrüger, versorge sie, unterstütze sie finanziell und das sei eben „ein klarer Verstoß gegen das Grundgesetzgebot, dem Wohl des deutschen Volkes zu dienen". 22 Die gegenwärtige Situation und die zunehmende Einwanderung erforderten es, das ebenso im Grundgesetz beschriebene Widerstandsrecht anzuwenden, das den Schutz der verfassungsmaßigen Ordnung verlange, wenn eine andere Abhilfe nicht möglich sei. Wie diese Widerstandspraxis dann aussehen soll, wird ebenso gesagt: Es sind Brandanschläge auf die Wohnheime von Asylbewerbern, die als grundgesetzlich abgedeckte und sogar geforderte Handlungen beschrieben werden. Ideologiekonform uminterpretierte Gesetzestexte sind es, die von

20 Zitiert nach Bosse, in: Recht und Wahrheit 3/4/90, S. 12.

21 Unabhängige Nachrichten (UN) 2/89, S. 6 f.

22 F. 53, S. 1, 2. 55f. 
den neonazistischen Autoren benützt werden, um der anvisierten Gewalttătigkeit den Anschein der Legalität zu geben. ${ }^{23}$

\section{Von der Macht der Massenmedien: die totalitäre Wirkungshypothese}

Den etablierten und gesellschaftlich anerkannten Medien, die man dann zitiert, wenn sie einem irgendwie nutzen können, begegnet man in neonazistischen Publikationen zumeist jedoch mit einem universal eingesetzten Manipulationsverdacht. Auch er schafft Entlastung und erlaubt es, die inhaltliche Auseinandersetzung mit den Urteilen der Öffentlichkeit zu umgehen: Manipulationen muss man eben nicht mehr diskutieren, man muss sich von ihnen nicht zu Reflexionen anregen lassen, sondern man kann sie, so die unter Neonazis verbreitete Devise, in ihrer Existenz bekămpfen. Denjenigen, die in den Massenmedien tätig sind, hălt man vor, sie verbreiteten "nichts als Gift" 24 oder eine "Giftsaat" ${ }^{45}$ man würde systematisch belogen und betrogen. Eine "gigantische Meinungsindustrie hämmert", schreibt ein Autor der Zeitschrift Die Bauernschaft, „gnadenlos die gewünschte ,Wahrheit" in die Gehirne kritikloser Massen".26 In dieser stetig wiederkehrenden Annahme, das eigene Volk sei Opfer einer perfiden Indoktrination und in der Verwendung von Giftmetaphern für den Vorgang der Informationsaufnahme, offenbart sich ein für die ideologische Gruppe typischer Manipulationsverdacht, den ich als ,totalitäre Wirkungshypothese' bezeichnen möchte. Behauptet wird nämlich eine umfassende und keinen Widerstand duldende Allmacht der Medien. Information wird verdinglicht und ist in diesem Verständnis kein Angebot für Nutzungsoperationen mehr, das man annehmen kann - oder eben nicht. Sie erscheint als Gift, als eine Substanz mit notwendig destruktiver Wirkung. ${ }^{27}$ Der Medienkonsument wird aus dieser Perspektive nicht als ein selbstständiges, eigenverantwortliches Subjekt gesehen. Er setzt den massenmedialen Einflüssen nicht seine individuelle Konstitution, seine Vorlieben und Interessen und seine besondere Biographie entgegen, sondern tritt ihnen im Zustand des hilflosen Ausgeliefertseins und der Unmündigkeit gegenüber. Er wird, so lautet die anthropologisch voraussetzungsvolle Annahme, in seinen Einstellungen und in seinem Verhalten durch offentlich vermittelte Deutungen des Realen determiniert. Eine solche totalitäre Wirkungshypothese hat für kleine extremistische Grup-

23 Siehe insgesant F. 53, S. 1-2.

24 Wehr' Dich!, 5/92, S. 16.

25 Haberer, in: Recht und Wahrheit 5/6/92, S. 10.

26 Bielenberg, in: Die Bauernschaft 4/91, S. 51.

27 Siehe hierzu kontrastiv die Ausfühnungen zum Prozess der Informationsaufnahme und dem Vorgang des Verstehens aus einer konstruktivistischen Sicht in: Foerster/ Pörksen, B. (1998), S. 97-100. 
pen gewisse psychologisch erklärbare Vorteile: Sie erlaubt es, die relative Wirkungslosigkeit der eigenen agitatorischen Anstrengungen auf eine wenig schmerzliche Weise zu entschuldigen. Sie muss dann nicht als selbstproduziert erscheinen, sondern lässt sich als Fremdverschulden ausgeben, das man entlarvt. Es sind die allmächtigen Medien und die Verschwörer, die die eigenen Bemühungen, andere Deutungen durchzusetzen, untergraben.

\subsection{Sprachliche und argumentative Muster}

\section{Umwertungen: Spielformen der Begriffsdestruktion}

Aus dem öffentlichen Diskurs über den Nationalsozialismus und den Neonazismus stammende Schlag- und Reizworter, die man gemäß der eigenen Perspektive der Weltbetrachtung für falsch bzw. für diffamierend hălt, werden in neonazistischen Publikationen aufgegriffen, sie werden umgewertet und ideologiegemaß richtiggestellt. Die Umwertung des gegnerischen Begriffs, die den Zweck verfolgt, den im Wort ausgedrückten Inhalt zu bezweifeln oder schlicht zu negieren, ${ }^{28}$ geschieht dabei graphemisch durch den Gebrauch von 'destruktiven Anführungszeichen'. Mit ihrer Hilfe sollen "Wörter ins Zwielichtige gerückt werden, eine ironische oder sogar pejorative Bedeutung" (Dieckmann 1964, S. 129) erhalten. Die Anführungszeichen fungieren in dieser Verwendungsweise als Signale der Infragestellung und Distanzierung, sie sind „das gewohnliche Mittel sprachpolitischer Illegitimitătserklärung von Wortgebrauchen" (Lübbe 1975, S. 108). So werden Worter wie Antisemit, Ausländerfeind, Ewiggestriger, Faschist, Nationalist, Neonazi, Rassist, Unbelehrbarer, Unverbesserlicherimmer wieder mit Anführungsstrichen versehen. ${ }^{29}$ Es sind die Feind- und Stigmaworter des Gegners, von denen man sich distanziert, deren Sachhaltigkeit man bezweifeln und negieren möchte. Auch der hăufige Kursivdruck derartiger Reiz- und Schlagworter, die Beifügung von so genannt oder angeblich gehoren zu den Distanzindikatoren, sie dienen der reaktiven Umwertung: Man benutzt sie zu der Bekundung von Ärger und Ablehnung. Sie sollen der Annahme Ausdruck verleihen, dass man die eigenen Auffassungen und Handlungen so nicht korrekt benennen kann.

$28 \mathrm{Zu}$ den verschiedenen Formen der destruktiven Umwertung gegnerischer Begriffe durch Anführungszeichen, Kursivdruck, die Beifügung von so genannt siehe Dieckmann (1964), S. 129ff.

29 Siehe exemplarisch F. 24, S. 1. 
Sprachthematisienungen in argumentativer Funktion: Abwehr der Inhalte Auch am Beispiel von argumentativ gebrauchten Sprachthematisierungen - das sind Sprachreflexionen, denen im Textzusammenhang die Funktion von Argumenten zukommt - ist der aufgebrachte und aggressive Umgang mit der gegnerischen Begrifflichkeit beobachtbar (zur Typologie siehe insgesamt Wengeler 1996). Die Reiz- und Schlagwörter, die die eigene Gruppe oder mit ihr assoziierte Einstellungen oder Handlungen in negativer Weise etikettieren, werden funktional eingeordnet, um sie inhaltlich zu diskreditieren. Weil sie, so die implizit oder explizit vertretene These, der Abwertung dienen oder die mit ihnen beschriebenen Sachverhalte nicht der Realität entsprächen, werden sie abgelehnt. Denkbewegungen und an einzelne Schlag- und Reizwörter gebundene Befürchtungen, die den gruppeninternen Konsens erschüttern, werden auf eine Weise kategorisiert, die nur erneute Belege für die Perfidie der Gegner liefern sollen. So heißt es in einer Schrift des Freundeskreises Freiheit für Deutschland, dass die Bezeichnung Ausländerfeindlichkeit sachlich unzutreffend sei, da man nichts gegen italienische Pizzabäcker oder in Deutschland arbeitende Schweizer Ärzte habe. Es handele sich um ein Propagandawort, das dem Ziel diene, ,jede Abwehrregung gegen die eindringenden betrügerischen fremdrassigen Invasoren zu verteufeln und zu kriminalisieren ". ${ }^{30}$ Die öffentlich verwendeten, die negativ gemeinten Zuschreibungen werden entsprechend durch ideologisch motivierte und argumentativ verwendete Sprachthematisierungen zurückgewiesen. Rechtsradikal und rassistisch gelten als Wörter aus dem „Schmähvokabular der Internationalisten ";31 die Zeitschrift Wikinger setzt sich mit den „uns so bekannten Totschlagwörtern wie ,Rassismus' und ,Ausländerfeindlichkeit" 32 auseinander; Die Bauernschaft attackiert die Parole Wehret den Anfängen als ein Schlagwort, das benutzt werde, um die eigenen Versammlungen zu storen. ${ }^{33}$ Man beruft sich, so wird hier deutlich, vor allem auf die vorausgesetzte bewusstseins- und handlungsbestimmende Macht derartiger Wörter und Parolen und ihre Benutzung als ein Mittel der Auseinandersetzung, um die gegnerische Sprache zu demontieren.

Selbstbeschreibung und Bekenntnis: sprachliche Ausweichmanöver Wohl um die Angriffspunkte für negative Zuschreibungen zu reduzieren, werden von Neonazis vielfach eher unverdächtige Selbstbezeichnungen

30 F. 60, S. 1, Z. $30 \mathrm{ff}$.

31 Vogel, in: Unabhängige Nachrichten (UN) 9/90, S. 4.

32 Wikinger $3 / 89$, S. 30.

33 Die Bauemschaft 2/92, S. 8. 
und Selbstbeschreibungen gewählt; auch bei der Verwendung von Grußund Abschiedsformeln, die ebenfalls Bekenntnischarakter haben, ist das Bemühen erkennbar, die eigene Gesinnung einerseits zu offenbaren und sie andererseits zu verbergen: Die zahlreichen sprachlichen Ausweichmanöver lassen sich als Versuche deuten, der schlagwortartigen Stigmatisierung als Nazi oder Neonazi zu entgehen. Neonazistische Gruppen etikettieren sich oftmals selbst als national oder nationalgesinnt oder auch, um gegenüber der hier bürgerlich genannten Rechten (gemeint sind etwa Parteien wie die Republikaner) noch eine sich im Gruppennamen manifestierende Distinktion aufrechtzuerhalten, als Nationalisten. ${ }^{34}$ Schon die Gruppennamen spiegeln die Neigung, eher integrative Selbstbezeichnungen zu wählen und nicht sofort durch eine allzu große weltanschauliche Eindeutigkeit potentielle Sympathisanten zu verschrecken. Sie heißen etwa Nationale Alternative, Nationale Liste, Hilfsorganisation für nationale politische Gefangene und deren Angehörige e.V. oder auch Nationalistische Front. Die explizite Selbstbezeichnung als Nationalsozialist beschränkt sich auf illegale und im Untergrund agierende Gruppierungen wie die Nationalsozialistische Deutsche Arbeiterpartei/Auslandsund Aufbauorganisation und prominente Einzelpersonen.

Ansonsten existieren graduell verschiedene Varianten der Offenkundigkeit; Selbstbeschreibungen und direkte oder indirekte Charakterisierungen des eigenen Standpunktes durch Gruß- und Abschiedsformeln, die offentlich fundamental diskreditiert sind, versieht man oftmals mit einem Moment des Fraglichen und eben nicht letztgültig Bestimmbaren: Manche Neonazis benutzen Anspielungen oder variieren das ideologisch ganz und gar eindeutige Bekenntnis - und schließen ihre Briefe „mit verbotenem Gruß “35 oder auch mit „Heil und Sieg!“, da die „Umkehrung dieses uralten deutschen Grußes", 36 wie es in einer ergănzenden Bemerkung zu einem Brief heißt, noch durch die Gesetze blockiert sei. Eine geheimsprachliche Komponente bekommt die Ideologiesprache der Neonazis, wenn Heil Hitler durch die Zahl 88 codiert wird; die beiden Zahlen stehen gruppenintern jeweils für den achten Buchstaben des Alpha-

34 So schreibt ein anonymer Autor in der Zeitschrift Aufbruch 11/91, S. 2: „Nationalismus ist für uns eine ganzheitliche Welt- und Lebensanschauung, auf der die politischen Forderungen gründen müssen. Hingegen gibt das Wort 'national' eine mehr gefïhlsbestimmte, ja oft diffuse und oberflächliche Haltung zu Volk und Land wieder. Tatsächlich können wir in Geschichte und Gegenwart eine Vielzahl von bürgerlichen und reaktionären Gruppierungen mit dieser Namensgebung feststellen. Nicht zuletzt von ihnen wollen wir uns durch den ,Nationalismus' absetzen."

35 Hellmund, in: Nachrichten der HNG 143/92, S. 10.

36 Kemper, in: Nachrichten der HNG 120/90, S. 16. 
bets. ${ }^{37}$ Auch die Schwärzung oder Auslassung eines Wortbestandteils, dessen Ausschreibung erst die letzte Eindeutigkeit stiften würde, ist beobachtbar. "Lieber Kamerad Dinter", so heißt es in der Zeitschrift Wehr' Dich!, „ich selber bin in der FAP und bin ein stolzer National..., und ich lese sehr viel über unsere gefallenen deutschen Soldaten". ${ }^{38}$ Die Zeitschrift Sieg fordert schließlich die "nationale und soziale Revolution".39 Man schreibt über "Nationale-Sozialisten" und "nationalen Sozialismus" 40 und nutzt, so lässt sich vermuten, die Auseinanderschreibung als ein Mittel, die sich, wenn eine Vereindeutigung aus irgendwelchen Gründen nicht von Vorteil wäre, immer noch zur formalen Distanzierung verwenden lassst (siehe auch Berning 1964, S. 137-142). In einem Artikel des Neonazis Thies Christophersen ist ein solcher Distanzierungsversuch nachweisbar: Er führt die abkürzende Etikettierung als Nazi auf das lexikalisierte Kompositum zurück, nimmt dieses auseinander, um sich dann durch den Rückzug auf die vergleichsweise eher unverdächtige Bedeutung der Wortbestandteile unangreifbar zu machen:

„Auf die Frage, ob ich denn heute noch ein ,Nazi' wäre, gebe ich folgende Antwort: Das Wort ,Nazi' ist heute zu einem Schimpfwort geworden, und auf Beschimpfungen gebe ich in der Regel keine Antwort. Wenn Sie mich aber fragen, ob ich heute noch ein Nationalsozialist bin, antworte ich: Das waren eigentlich zwei Fragen. Einmal ob ich national bin - und ob ich sozial bin. Ich gebe mir Mühe, beides zu sein. Ob mir das immer gelingt, weiß ich nicht. “41

\section{Umdeutung: ein Beispiel für die Besetzung von Begriffen}

Gemeinsprachlich, so lässt sich zeigen, bezieht sich die Rede von einem Völkermord häufig auf die Verbrechen der Nationalsozialisten. ${ }^{42}$ Schlagwortartig gebraucht wird das Wort dagegen in neonazistischen Publikationen primär, um das gefürchtete Ergebnis der Einwanderung und des Zusammenlebens mit Ausländern zu bezeichnen und einen vermeintlich

37 Siehe Bachem (1983), S. 78.

38 Wehr' Dich!, 1/90, S. 17.

39 Deckert, in: Sieg 2/89, S. 8.

40 Aufrecht!, S. 3 und Wendt, in: Aufrecht!, S. 12.

41 Christophersen, in: Die Bauemschaft 4/90, S. 23.

42 Indiz für diese Verwendung ist etwa die Debatte der SPD-Politiker Glotz und Duve, die sich mit der Frage befasste, ob der Begriff Völkermord ausschließlich für die NS-Untaten gelten solle: Duve hatte im Juli 1992 in einem Zeitungsartikel die serbisch-jugoslawische Armee für Völkermord-Handlungen verantwortlich gemacht und Glotz argumentierte in einer Replik, dass der Begriff Völkermord nur für die Kennzeichnung von NS-Verbrechen verwendet werden solle. Siehe Duve (1994), S. $128 \mathrm{ff}$. 
in der Gegenwart sich vollziehenden Völkermord an den Deutschen zu geißeln. Es kursieren Aufkleber mit der Textzeile „Rassenmischmasch = Völkermord“, und man behauptet, das eigene Volk solle in einem "multirassischen Völkerbrei“43 ausgelöscht werden. "Jede Integration ethnischer Fremdgruppen“, so eine Zeitschrift, ,ist als Völkermord klar definiert." 44 Das heißt: In den Veröffentlichungen neonazistischer Gruppen wird das Wort Völkermord aus seinem gemeinsprachlich konventionalisierten Gebrauchszusammenhang herausgelöst; man deutet es um, transformiert es in ein ideologiespezifisches Schlagwort, indem man unter dem Lexem Mord, das Bestandteil des Kompositums ist, nicht mehr die gewaltsame Vertreibung von Gruppen oder die Tötung von Menschen versteht, sondern das Resultat von Rassenmischung und die Vernichtung einer rassisch definierten Identităt (zum Schlagwort siehe umfassend Kaempfert 1990). Diese Umdeutung ist es, die das Schlagwort Völkermord in das Zentrum der spezifischen Geschichtsverdrängung und -verleugnung hineinrückt. Es ist ein Zeugnis der offensiven Selbststilisierung als Opfer.

\section{Okkupation von Schlüsselwörtern und Schlüsselszenen:}

\section{Neukontextualisierung}

Die Verbrechen der Nationalsozalisten gelten in der Öffentlichkeit als beispiellos, völlig neuartig, einmalig, einzigartig und singulär (siehe Römer 1990, S. 260f.). Niemals in der gesamten Geschichte sei ein einzelner Bevölkerungsteil - Männer, Frauen, Kinder, Gesunde und Kranke - isoliert worden, um "mit allen nur möglichen staatlichen Machtmitteln" (Jäckel zit. nach Lipstadt 1996, S. 332) umgebracht zu werden. Es handele sich um ein „völlig neuartiges Verbrechen gegen Rang und Stand der Menschheit" 45 - kurzum: der nationalsozialistische Völkermord entziehe sich jedem Vergleich.

Neonazistische Gruppen agitieren gegen diese Behauptung der Unvergleichbarkeit, indem sie Einschätzungen dieser Art reaktiv neu kontextualisieren. Als das weltgeschichtlich "einmalige Verbrechen" 46 gilt ihnen nicht der (von ihnen geleugnete) Holocaust, sondern die Behandlung von Deutschen in den Kriegsgefangenenlagern der Alliierten nach 1945. Man fordert die Alliierten auf, „zu bekennen, dass hier von ihrer Seite ein Völkermord begangen wurde“, 47 dass in den „allierten $\mathrm{KZs}$ " - gemeint

\footnotetext{
43 F. 104, S. 2, Z. 75.

44 Wikinger 2/92, S. 21.

45 Zitiert nach Römer (1990), S. 260.

46 Bayrich, in: Leitheft 42, S. 9.

47 Wikinger 2/92, S. 19.
} 
sind die Gefangenenlager - eine Million „deutsche Menschen diesem Völkermord zum Opfer gefallen "48 seien. Die Beschreibungen der Zustände in diesen Lagern, in denen ein furchtbarer Hunger geherrscht habe, wirken oftmals wie Zitate aus den Berichten von Häftlingen der nationalsozialistischen Konzentrationslager. Beispielhaft sei eine Schilderung zitiert. Über den "Massenmord an deutschen Kriegsgefangenen" heißt es hier:

„Die Leichen der Verhungerten wurden täglich auf Karren weit außerhalb der Lager in vorbereitete lange Gruben gekippt und in fünf Lagen und langen Reihen aufgeschichtet. Nach Verfïllung mit dem zuvor ausgebaggerten Erdreich erfolgte die Planierung der Massengräber.“49

Überhaupt sind die Versuche, sich des Diskurses über den Völkermord der Nationalsozialisten zu bemächtigen und die entsprechenden Schlüsselworrter und -sequenzen als provozierende Wirkungspotentiale zur Diskreditierung von Gegnern zu verwenden, ziemlich umfassend (zur Definition des Schlüsselwortes siehe Bachem 1979, S. 63). So verlangt man "für die Hinterbliebenen Wiedergutmachung" und fordert, "dass auf dem Lagergelände eine würdige Gedenkstätte entsteht" 50 - nützt hier also zentrale Begriffe des historischen und moralischen Diskurses über die NS-Zeit, um die Täter und die Deutschen insgesamt als die eigentlichen Opfer erscheinen zu lassen. Der „wirkliche Holocaust"51 habe nämlich erst nach dem Kriege in diesen Lagern stattgefunden. Allerdings sind derartige NS.Vergleiche und die Hinweise, die sich in irgendeiner Weise auf die nationalsozialistischen Untaten beziehen, in der Regel von besonderer Art: Man verwendet - einerseits - das gesamtgesellschaftlich diskreditierte Vokabular der NS-Zeit (z.B. Endlösung, KZ, Sonderbehandlung) in vielfältiger Weise zur Diffamierung, teilt aber - andererseits - aus ideologischen Gründen nicht die Verurteilung des Nationalsozialismus, die mit solchen Analogieverweisen oder Gleichsetzungen in der Regel einhergeht (zum NS-Vergleich als Mittel der politischen Auseinandersetzung siehe Stötzel (1995), S. 369-381). Neonazis sprechen von der „Endlösung' für das deutsche Volk"52 und der "Sonderbehandlung unserer politischen Gefangenen". ${ }^{53}$ Sie nutzen also das diffamierende Wirkungspotential eines spezifisch und neu kontextualisierten NS-Vokabulars - und zwar ohne dass sie mit der allgemeinen Verurteilung des

\footnotetext{
48 Wikinger 2/92, S. 18.

49 F. 10, S. 2, Z. $46 \mathrm{ff}$.

50 Wiktnger $2 / 92$, S. 19.

51 F. 10, S. 1, Z. 2.

52 Tele-Blitz 3/92, S. 4.

53 Müller, in: Nachrichten der HNG 149/93, S. 6.
} 
Nationalsozialismus übereinstimmen. Sie streichen vielmehr seine vermeintlich positiven Seiten heraus.

\section{Gegenschlagwörter: Konterkarierung von Vorwürfen}

Eine weitere Möglichkeit, der als gegnerisch wahrgenommenen Sprache ihre Wirkung zu rauben, besteht im Gebrauch von ,Gegenschlagwörtern' (Hannappel/Melenk 1990, S. 259). Sie sollen eine behauptete Realität ideologiegemäß erschließen - und werden direkt und offensichtlich gegen andere Reiz- und Schlagwörter, die für falsch gehalten werden, gesetzt, um die Wahrnehmung des zur Debatte stehenden Problems umzuorientieren. Kandidaten für derartige Gegenschlagwörter sind Vokabeln wie Antigermanismus (versus Antisemitismus) oder Deutschenhass und Inländerhass (versus Ausländerhass), Deutschenhetze (versus Volksverhetzung), fremdtümelnd (versus deutschtümelnd), Inländerfeindlichkeit (versus Ausländerfeindlichkeit), Internazi (versus Nazi) und nationalmasochistisch (versus nationalsozialistisch). Auch das außerordentlich häufig gebrauchte Adjektiv deutschfeindlich und die substantivischen Varianten Deutschenfeindlichkeit und Deutschfeindlichkeit haben in verschiedenen Publikationen den Status von Gegenschlagwörtern. Sie werden verwendet, um auf den höchst unliebsamen Vorwurf der Ausländerfeindlichkeit $\mathrm{zu}$ reagieren. Ein Beispiel illustriert, das Gegenschlag. wörter immer direkt und oftmals schon in einem einzigen Satz gegen das entsprechende Reiz- oder Schlagwort der anderen Seite gesetzt werden; so kritisiert ein Autor, dass der Bundespräsident die Schirmherrschaft einer Demonstration übernommen habe, die sich gegen Ausländerfeindlichkeit richtet. In den anschließenden Sätzen liest man:

„Warum demonstriert niemand gegen Deutschfeindlichkeit? Man will uns zwingen, Rauschgifthändler, Messerstecher, Straßenräuber und Sozialbetrüger lieb zu haben, nur weil sie Ausländer sind. Andernfalls werden wir als Rechtsradikale oder als Nazis beschimpft. Dabei sind wir Deutsche allen Fremden gegenüber aufgeschlossener als jedes andere Volk. Nein, wir sind keine Ausländerfeinde, aber unsere Regierung ist absolut deutschfeindlich! Das ist unser Problem!"54

Was hier anvisiert wird, ist nicht weniger als eine Umorientierung der öffentlichen Wahrnehmung; Demonstrationen gegen ausländerfeindliche Ausschreitungen gelten den neonazistischen Gruppen, vergleichbar mit Gedenktagen und Denkmälern, die an die Schrecken des Nationalsozialismus erinnern, als Reflexionsanlässe der Öffentlichkeit, die man gemäß der eigenen Perspektive der Weltbetrachtung - ablehnt, kritisiert oder auch durch Aufrufe zu eigenen Kundgebungen konterkariert. So

54 F. 103 , S. 1, Z. 14 ff. 
findet sich in den Veröffentlichungen von Neonazis und anderen rechtsextremen Gruppen immer wieder die Forderung, man solle eben gerade eigene deutschfreundliche Sympathiekampagnen und Lichterketten initiieren. Erwünschter Anlass der Trauer und des ideologiespezifischen Erinnerns und Gedenkens sind beispielsweise die „Opfer der Multikultur" 55

\section{Karikaturen des Religiösen: Verehrung und Verspottung}

Die Sprache der Neonazis ist durchsetzt mit religiösen Formeln und Vergleichen, die dazu dienen, die eigenen Aktivitäten in der Welt durch das verwendete Vokabular zu heiligen. ${ }^{56}$ Man gebraucht sie, auch das ist eine Parallele zum pseudoreligiösen Charakter der nationalsozialistischen Ideologie, zur Legitimierung der eigenen Ziele, setzt sie gezielt als Mittel der Persuasion ein. Immer wieder nimmt man auf einen wohl personal gedachten oder auch auf einen in der Natur vermuteten bzw. mit ihr gleichgesetzten Gott Bezug, behauptet, die eigenen Gesinnungen und Taten stünden im Einklang mit dem Walten göttlicher Mächte und versieht sie auf diese Weise mit einer Art metaphysischer Weihe. Wohl um die innerweltlich ausgerichtete Sakralisierung der eigenen Standpunkte einer möglichst großen Anzahl von Lesern nahezubringen und sie auf eine unmissverständliche Weise bewusst $z \mathfrak{u}$ machen, ist das $z \mathfrak{u}$ diesem Zweck verwendete Vokabular relativ unspezifisch. Man beendet Flugblätter und -schriften mit Appellen (und Aufrufen zur Gewalttätigkeit) wie „Deutsches Volk: Hilf Dir selbst, dann hilft dir Gott! “57 oder einem unvermittelt hinzugefügten „Das walte Gott! Amen!“,58 erklärt die perzipierte Bedrohung des Volkes zu einem "Sakrileg am Leben auf dieser Erde" 59 oder zu einem Verstoß gegen „die ewigen Gesetze der Natur und damit Gottes"60 - und diabolisiert entsprechend die Gegner und ihre Gesinnung. Sie erscheinen als Manifestationen des Teuflischen und Satanischen, ${ }^{61}$ des Bösen und als "Ausgeburten der Hölle".62 Kurzum: Es ist die Tendenz erkennbar, den oftmals nicht näher spezifizierten und religiös nicht weiter ausgedeuteten Gegensatz von Gott und Teufel mit

55 F. 95, S. 1, Z. 24.

56 Trotz dieser Tatsache ist in vielen Texten ein tiefsitzendes antichristliches Ressentiment nachweisbar, dass auf die These zurückgeht, das Christentum verweichliche, stifte zu einem unnützen Mitleid mit den Schwachen und Benachteiligten an.

57 F. 31, S. 2, Z. 96.

58 F. 27, S. 2, 2. 68.

59 Gärtner, in: Unabhängige Nachrichten (UN) 2/90, S. 5.

60 Wikinger $1 / 89$, S. 8.

61 Roeder, in: Deutsche Bürgerinitiative e. V. - weltweit 4/92, S. 2.

62 F. 101, S. 2, Z. 42. 
der Unterscheidung von Eigen- und Fremdgruppe zur Deckung zu bringen.

Aber das religiöse Vokabular dient nicht allein dazu, das eigene Tun zu verklären, einen offenbar erkennbaren göttlichen Willen für konkrete ideologische Ziele anzurufen und den Gegner zu verteufeln, sondern auch dazu, Abgelehntes als irrational zu verspotten. Metaphorisch wird Sprache aus dem religiösen Bereich und der Sphäre des Glaubenskampfes insbesondere dann verwendet, wenn die Faktizität des Holocaust bestritten und die eigenen Auffassungen und Handlungen zu einem Martyrium für die gerechte Sache überhöht werden sollen. Im intratextuellen Kontext fungieren Wörter wie Glaube und Religion, Dogma und Heiligkeit dann als Metaphern, die man einsetzt, um die Protagonisten des öffentlichen oder fachspezifischen Diskurses über den Holocaust zu diskreditieren und um der Judenvernichtung metaphorisch ein Moment des Fraglichen und Zweifelhaften $z u$ attribuieren, das sich in den Texten letztlich immer zu der Generalthese, diese habe überhaupt nicht stattgefunden, steigert. Personen, die sich mit Fragen der Judenvernichtung befassen, nennt man - je nach Rang und öffentlicher Geltung - Päpste oder Kirchendiener. Man attackiert einen "Gaskammerkult" ${ }^{43}$ und bezeichnet die Gaskammern von Auschwitz als den „heiligsten Schrein der Welt". ${ }^{64}$ Auschwitz sei ein neues Golgatha ${ }^{65}$ - der zentrale Kultort einer Gemeinschaft, die an die Holocaustreligion ${ }^{66}$ glaube. Man spricht von den "geheiligten 6 Millionen" - und bezieht sich auf die Zahl der Opfer, nennt Anne Frank in polemischer Absicht eine „Heilige" 67 und vertritt die Auffassung, zahlreiche Menschen würden mit "unbedingtem religiösen Fanatismus"68 an den Holocaust glauben.

Die Umdeutung des Holocaust $z \mathfrak{u}$ einem mit dogmatischem Eifer verfochtenen Glaubensartikel führt aber nicht dazu, dass man auch den eigenen Standpunkt als schlichten Glauben neben vielen möglichen und eventuell ebenso als Dogma interpretiert. Fanatiker und Eiferer sind allein die Gegner. Man selbst klärt auf. Zwar behält man gelegentlich die metaphorisch eröffnete Perspektive bei und appelliert an die Religionsfreiheit, ${ }^{69}$ aber das geschieht nur, um sich polemisch das Recht zu erstreiten, die eigentliche Wahrheit endlich zu verkünden: Nur ein einziges Mal wird in den untersuchten Texten die verfochtene Holocaustleugnung als

\footnotetext{
63 Honsik, in: Halt 46/88, S. 1.

64 Graf, in: Kritik - Die Stimme des Volkes 84/93, S. 99.

65 Vogt, in: Die Bauernschaft 3/93, 13.

66 Vogt, in: Die Bauernschaft 3/93, 13.

67 Vogt, in: Die Bauernschaft 3/93, 11.

68 Graf, in: Kritik - Die Stimme des Volkes 84/93, S. 107.

69 Remer-Depesche 3/93, S. 1.
} 
bedauerlicherweise strafbarer Gegenglauben ${ }^{70}$ etikettiert. Die Regel ist, dass man sich selbst nicht als einen Andersgläubigen begreift, sondern sich im Falle der Strafverfolgung zum Opfer eines brutalen und mit dogmatischer Härte verfochtenen Kampfes stilisiert. Man sieht sich der Inquisition und politischen Hexenprozessen ${ }^{71}$ ausgesetzt und erhebt sich regelhaft zu einem Ketzer, ${ }^{72}$ der allein und voller Mut, die Wahrheit sagt . und eben deshalb verfolgt wird.

\section{Bilder des Krieges: Konstruktion von Notwehrsituationen}

Militärische und kriegerische Metaphorik eignet sich insbesondere zur Schwarz-Weiß-Zeichnung und zur Konstitution von Gegensätzen und harten Kontrasten. Man evoziert die Vorstellung klarer Frontverläufe, setzt in jedem Fall die Unterscheidung von Freund und Feind und die Existenz polarer Positionen. ${ }^{73}$ Zur klassischen Konstellation der kriegerischen Auseinandersetzung, die metaphorisch genutzt wird, gehören zwei Parteien, unvereinbare Interessen, ein Schlachtfeld, der bewaffnete Kampf und die Verfolgung bestimmter Ziele und Strategien zur Vernichtung des Gegners. Konstruktive Zusammenarbeit und die Möglichkeit zur friedlichen Kompromissfindung liegen im toten Winkel dieser Metaphern. In den neonazistischen Publikationen werden als gegnerisch interpretierte Handlungen, Standpunkte und Interessen als Bestandteil von kriegerischen Auseinandersetzungen begriffen. „Der Schießkrieg ruht", schreibt ein Autor, „aber der Propagandakrieg tobt" ${ }^{74}$ Themen, mit denen man sich befasst und Gegenwartstendenzen, die die ideologiegesteuerte Weltwahmehmung irritieren, gelten als Fronten. Ein Autor sieht sich in einen „Fünf-Fronten-Krieg“75 verwickelt. Er nennt: „die Kriegsverbrecherfront“, die „Holocaust'-Front“, „die Überfremdungsund Asylschwindelfront", "die Front zur Unterdrückung jeden Nationalgefühls" und schließlich „die Begriffsfront zur Auslöschung aller nationaldeutschen Begriffe und Worte ". ${ }^{76}$ Was man ablehnt, wird jedoch nicht nur allgemein als Krieg, und wogegen man angehen möchte, wird nicht nur als Front, sondern auch als zielgerichtet eingesetzte Waffe beschrieben. So gelten Abtreibung, Emanzipation, Homosexualität, die multikulturelle Gesellschaft und zahlreiche weitere Phänomene als Ausrottungs-

70 Germanicus, in: Die Bauernschaft 1/92, S. 13.

71 Halt $48 / 89$, S. 4.

72 Ochensberger, in: Sieg 2/89, S. 3.

73 Siehe insgesamt Küster (1978) und auch (1989), S. 87.

74 Sieg $9 / 90$, S. 9.

75 Sieg $9 / 90$, S. 9.

76 Sieg $9 / 90$, S. 9. 
waffen zur Zerstörung des Volkes. ${ }^{77}$ Gegen diese offensiv eingesetzten Werkzeuge der Destruktion bringt man als eine "Verteidigungswaffe"78 den Appell zum Kinderkriegen ins Spiel: Junge Frauen sollten Kinder gebären, um die imaginierte Ausrottung der Deutschen zu verhindern. Hintergrund dieser und ähnlicher Vorstellungen ist vermutlich die Ursprungsmetapher der Nationalsozialisten von einem sich vollziehenden Geburtenkrieg. ${ }^{79}$ Immer wieder wird betont, dass man sich "faktisch in einer Art Kriegszustand mit den herrschenden Mächten" 80 befinde. Massen von Menschen werden als Heere oder Truppen beschrieben; die Flucht von Menschen aus ihrem Herkunftsland und der Prozess der Immigration erscheinen den neonazistischen Autoren regelhaft als Invasion und als „Fortführung des Krieges gegen das Deutsche Reich mit der Ziel der Vernichtung". ${ }^{81}$ Ausländer gelten demgemäß als Invasoren oder Invasionstruppen. ${ }^{82}$ Man sieht einen totalen Krieg und eine "letzte große Schlacht gegen den wehrlosen deutschen Menschen "83 heraufziehen. Die Metaphorik stammt aus dem Bereich der konventionellen Kriegsführung; die moderne, hochentwickelte Kriegstechnologie der Atomraketen, Sprengköpfe und Mittelstreckenraketen wird in keinem Fall metaphorisch genutzt. Man hat es mit Fronten, Schlachten und dem gezielten Einsatz nicht näher spezifizierter Waffen zu tun. ${ }^{84}$ In den Zeiten der Gefahr und des Getümmels, die hier evoziert werden, scheint kaum Zeit zur Reflexion. Die verwendeten Kriegsmetaphern drängen zur unmittelbaren Reaktion, sie eignen sich zur Mobilisierung, zum Aufgebot letzter Kräfte. ${ }^{85}$ Sie setzen Szenarien der Gefahr ins Bild, die keine Nachdenk. lichkeit und kein Zaudern mehr gestatten und verlangen, wie es beispielhaft in der folgenden Textpassage zum Ausdruck kommt, nach der raschen Intervention:

„Bonn hat uns den Krieg erklärt. Bonn hat sich mit den Millionen Horden fremdländischer Invasoren gegen das deutsche Volk verbündet. Bonn wirft Millionen von Eindringlingen in die Schlacht gegen das deutsche Volk. [...] Deutsche wehrt euch, wehrt euch, wehrt euch. Es ist Notwehr. Es ist Pflicht. Es ist kein Ausländerhass. Begehrt auf. Sich laut zu Wort zu melden, ist Notwehr." 86

77 Siehe insgesamt F. 21.

78 F. 21, S. 4, Z. 189.

79 Siehe Berning (1964), S. 86f.

80 Aufbruch 11/91, S. 6.

81 Germanicus, in: NS-Kampfruf 79/89, S. 6.

82 F. 67, S. 1, Z. 10 und F. 55, S. 2, Z. 89.

83 Stoß, in: Remer-Depesche 5/92, S. 1.

84 Siehe Baldauf (1997), S. 237.

85 Siehe auch Sontag (1989), S. $12 f$.

86 Remer, in Remer-Depesche 4/92, S. 4. 
Aufschlussreich und von exemplarischer Bedeutung ist, in welchem Ausmaß mit Hilfe der verwendeten Metaphern, die von einer Kriegserklärung, der Invasion und der Schlacht handeln, der Aggressionscharakter der Feindseite betont wird. Es handelt sich um eine Offensivmetaphorik, die es möglich macht, Situationen der Notwehr zu konstruieren und die eigenen Handlungen stets und mit Hilfe einer zu diesem Zweck eingesetzten Defensivmetaphorik als Maßnahmen des Widerstands darzustellen. ${ }^{87}$ Man wird angegriffen - und reagiert. Man droht überrannt zu werden - und soll (man beachte die stereotyp wiederholte Parole „Wehrt euch!") augenblicklich zur Gegenattacke übergehen. Das Geschehen wird auf eine Weise interpunktiert, die es gestattet, die eigene Seite als Opfer und die Handlungen der Gesinnungsgenossen als situationsadäquate Reaktionen erscheinen zu lassen. Man kann hier die metapherngestützte Konstitution eines eigenen Rechts- und Unrechtsbewusstseins beobachten: Die Metapher des Angriffs und des Krieges erlaubt es, von einer Ausnahmesituation auszugehen, in der die eigenen Maßnahmen stets als die Folge und nicht die Ursache von Aktivität angesehen werden können. Es ist die Wirklichkeit des Opfers, das sich nur wehrt, und der Zwang der Situation, der hier als Begründung geltend gemacht werden. Wenn man den Akt der Notwehr nicht mehr, wie in diesem Beispiel, metaphorisch als rhetorische Gegenwehr und den Einsatz des Wortes begreift, wird mit einer derartigen Interpunktion des Geschehens auch reale, das heißt ganz und gar unmetaphorisch gemeinte Gewalt legitimierbar. 88

\section{Reaktive Neubildungen: Schaffung von Bezeichnungsvarianten}

Nicht immer steht das passende Wort zu Verfügung, wenn man eine missliebige Sicht der Dinge konterkarieren möchte, um dann vielleicht nicht nur mit den Mitteln der Sprache, sondern auch mit Gewalt - gegen sie vorzugehen. Zahlenmäßig sehr kleine Gruppen, deren Weltsicht im Widerspruch zu jener der Mehrheitskultur steht, müssen vielmehr ihre jeweiligen Interpretationsvokabeln und Bezeichnungsvarianten oftmals erst neu bilden, um ihrer Ideologie sprachlich Geltung zu verschaffen. Ein möglicher Grund ist, dass sich womöglich ein Widerspruch zwischen

87 Es lässt sich am Beispiel dieses Textes somit eine Beobachtung bestätigen, die Küster (1989, S. 88) gemacht hat: Küster selbst spricht von „militärischen Metaphern“, die den „Aggressionscharakter der kommentierten Gegenstände“ akzentuieren. Diesen stünde „auf der anderen Seite eine semantisch positive Defensivmetaphorik" gegenüber.

88 Tatsächlich taucht die unmetaphorisch gemeinte Rede von einer dringend gebotenen Selbstverteidigung oder einem Akt der Notwehr in zahlreichen Schriften auf, wenn es darum geht, Gewalt zu rechtfertigen. 
ihren kommunikativen Bedürfnissen und dem vorhandenen sprachlichen Material ergibt - und dass dieser Widerspruch eben durch die Bildung von Neologismen ausgeräumt werden kann (zum Neologismus insgesamt siehe Herberg/Kinne 1998).

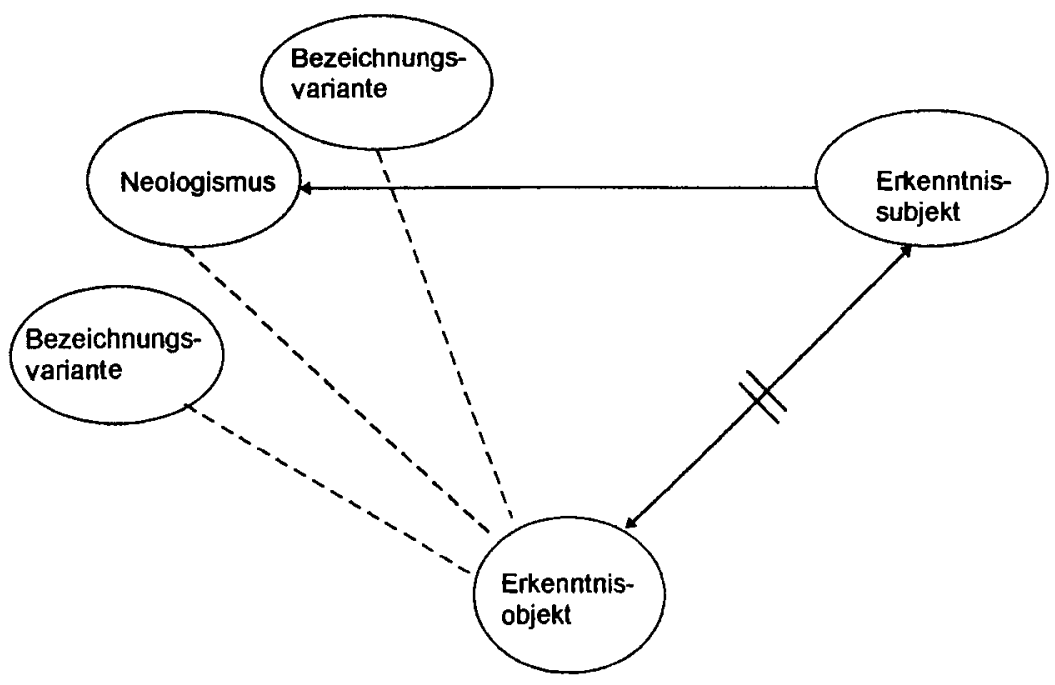

Der Erkenntnisvorgang ideologischer Gruppen, der zur Bildung von Neologismen führt, lässt sich modellhaft in drei aufeinander bezogene Elemente zergliedem. Diese sind: ein erkennendes Subjekt (hier: der Einzelne als Mitglied einer ideologischen Gruppe), ein Erkenntnisobjekt (ein Sachverhalt, auf den man sich bezieht) und eine im neuen Wort enthaltene Aussage über die angebliche Beschaffenheit des Erkenntnisobjektes, mit der andere Deutungen des Wirklichen konterkariert werden sollen. Angenommen wird: Einzelne Mitglieder ideologischer Gruppen erkennen Wirklichkeit in Relation zu den Prämissen, die in ihrer Ideologie angelegt sind. Sie besitzen also, wie andere Beobachter auch, keinen direkten und unverstellten kognitiven Zugriff auf die Welt der Erkenntnisobjekte. Sie setzen sich mit ihr auseinander - und versuchen, andere Bezeichnungsvarianten zum Zweck der Persuasion mit den ideologiesprachlich schon vorhandenen Tendenzwörtern zu attackieren. Nun kann es, so meine Hypothese, zu der Situation kommen, dass dies aus irgendeinem Grund nicht gelingt; das vorhandene Sprachmaterial erscheint nicht effektiv oder schlicht nicht ausreichend. Die mögliche Folge: Das gewünschte Benennungshandeln ist in einem ersten Stadium für eine gewisse Zeit blockiert, da die Tendenzwörter zur Auseindersetzung fehlen oder die eventuell vorhandenen - warum auch immer - nicht verwendet werden; gleichzeitig sind es die Bezeichnungsvarianten der gegnerischen Seite, die offensichtlich in besonderer Weise als unerträglich und provozierend empfunden werden. Man möchte sie kommentieren, demontieren, richtigstellen, sie nicht stehen lassen; und dieses kommunikative Bedürfnis, das seine Ursache in der Erfahrung ideologischer und weltanschaulicher Differenz hat, führt zur Suche nach einer passenden sprachlichen Form - und schließlich zur Bildung von Neologismen, die gegen die vorhandenen Bezeichnungsvarianten ins Feld geführt werden. Sie sind den Interessen ihrer Benutzer dienlich und sollen Deutungen und Prioritäten bei den Adresssaten durchsetzen oder bestärken. 
Das Grundmuster, das solchen Neuschöpfungen in den untersuchten neonazistischen Medien offensichtlich zugrundeliegt, ist denkbar einfach: Einzelne Schlüsselwörter des historischen und moralischen Diskurses über die NS-Zeit werden von den neonazistischen Autoren wahrgenommen und erlangen aus ihrer Sicht den Status von Reizwörtern, die Demontageversuche provozieren und die verbale Energie auf sich lenken. So werden - um nur ein Beispiel zu nennen - immer wieder in der Öffentlichkeit diskutierte Zahlenangaben, die von den Opfern der NS.Verbrechen handeln, als Bestimmungswörter ideologiesprachlicher Komposita benutzt. Das Grundwort Lüge soll dann jeweils signalisieren, die Opferzahl sei falsch, sie sei das Ergebnis von Manipulationen, die man vielfach durch stereotype antisemitische Behauptungen (der angebliche Wunsch von Juden, sich zu bereichern, Wiedergutmachungszahlungen zu erpressen oder aber die Weltherrschaft zu erlangen) erklärt. Beispiele für derartige Neulexeme, die die Aussagen über die Zahl der Opfer der in Auschwitz Ermordeten unglaubwürdig machen sollen, sind Komposita wie 6Millionen-Lüge, 4-Millionen-Auschwitz-Lüge oder auch 1-1,5-MillionenLüge. ${ }^{89}$ Andere Neologismen der neonazistischen Ideologiesprache dienen nicht mehr dazu, die Zahl der von den Nationalsozialisten Umgebrachten anzuzweifeln, sondern sie werden gebraucht, um ihre Ermordung insgesamt $z u$ leugnen. Sie verweisen etwa auf das größte nationalsozialistische Konzentrationslager, das zum Symbol für das Universum der nationalsozialistischen Vermichtungslager geworden ist: Auschwitz. Entsprechend gehäuft treten Neologismen auf, mit denen man die Faktizität der Morde in diesem Lager und mehr oder minder deutlich immer wieder auch die Ermordung der Gefangenen und Verschleppten insgesamt als bloße Phantasmagorie zu entlarven trachtet (2.B. Auschwitz-Lügner, Auschwitz-Mythos, Auschwitz-Schwindel). 90 Eine weitere Gruppe von neu gebildeten Komposita bezieht sich auf die - von Neonazis angezweifelte bzw. geleugnete - Art und Weise der Tötung in den als Duschräumen getarnten Gaskammern (z.B. Gaskammerlüge, Gaskammer-Schwindel, Vergasungslüge) ${ }^{91}$ oder auf den Holocaust insgesamt (z.B. Holocaust-Betrug, Holocaust-Legende, Holocaust-Lüge, Holocaust-Schwin-

89 Siehe in dieser Reihenfolge: Drack, in: Die Bauemschaft 4/90, S. 55; F. 99, S. 1, Z. 12; Drack, in: Die Bauernschaft 4/90, S. 55.

90 Siehe in dieser Reihenfolge: F. 25, S. 1, Z. 2; F. 25, S. 1, Z. 31; Fikentscher, in: Die Bauernschaft 2/89, S. 60.

91 Siehe in dieser Reihenfolge: F. 25, S. 1, Z. 34; Eidgenoss 5/6/89, S. 5; Kiwisch, in: Sieg $4 / 5 / 89$, S. 22.

92 Siehe in dieser Reihenfolge: F. 98, S. 2, Z. 66; Graf, in: Kritik - Die Stimme des Volkes 84 , S. 97 ; F. 98, S. 2 , Z. 72 ; Halt $55 / 90$, S. 1. 
del) ${ }^{92}$ Neulexeme wie Holocauster, Holocaustler und auch Exterminationist - eine abwertend gebrauchte Gegenbildung zu der Selbstbezeichnung als Revisionist - sollen schließlich jene Menschen summarisch bezeichnen und zu einer feindlichen Gruppe zusammenfassen, die die Massenvergasung nicht abstreiten und den Holocaust nicht leugnen. ${ }^{93}$ „Exterminationisten“", heißt es in einer Broschüre, „sind Holocaust-Gläubige, die dieses Verbrechen für eine Realität halten". ${ }^{94}$ Was von ihnen gesagt wird, nimmt man nicht inhaltlich wahr, man diskutiert es nicht, sondern ordnet die Äußerungen der zum Gegner erkorenen Anderen pauschal durch Neologismen wie Auschwitz-Propaganda, Holocaust-Propaganda oder Holocaust-Hetze ein. ${ }^{95}$

Man bietet, so wird an den geschilderten Einzelbeispielen erkennbar, ganze Ausdrucks- und Begriffsnetze an, um den gegnerischen Auffassungen etwas entgegenzustellen, sie mit den eigens kreierten Bezeichnungsvarianten deutend und wertend $z u$ überziehen und den Gefühlen der Abwehr and Ablehnung die passende sprachliche Form und die Möglichkeit der Entäußerung zu geben. Die zahlreichen Gegenbildungen offenbaren, dass eine Loslösung von der geistigen und sich eben auch in Sprache manifestierenden Deutungshoheit des Gegners nicht geglückt ist: Auch in der abwertenden Kommentierung und im Versuch der Demontage taucht das jeweils Abgelehnte wieder auf, gelangt - wenn auch natürlich unter negativen Vorzeichen - erneut ins Zentrum der Aufmerksamkeit. Man muss darüber sprechen, was die anderen, die Gegner, denken. Was sich am Beispiel dieser Kopplung an das Wertesystem des Gegners beobachten lässt, ist ein Phänomen, das hier, reaktive Sprachproduktivität $t^{\prime}$ genannt wird. Mit diesem Begriff soll ein sich sprachschöpferisch auswirkender Kausalzusammenhang angedeutet werden, der sich folgendermaßen skizzieren lässt: Je relevanter eine Vorstellung in der öffentlichen Wahrnehmung ist und je größer die Differenz dieser Vorstellung zu der Auffassung der ideologischen Gruppe, desto eher wird diese Gruppe versuchen, durch Propagierung von ideologiespezifischen Konkurrenzausdrücken und eben auch durch die Bildung von Neologismen eine Wahrnehmungskorrektur zu erreichen. Man kann nämlich davon ausgehen, dass die ideologische Gruppe durch die öffentlich verbreitete Vorstellung ihr Interesse tangiert und verletzt sieht, eigene Deutungen zur Geltung zu bringen und schließlich durchzusetzen. Und es ist

93 Siehe in dieser Reihenfolge: Honsik, in: Halt 46/88, S. 1; Remer-Depesche 3/93, S. 4;

Vogt, in: Kritik - Die Stimme des Volkes 75/92, S. 8.

94 Vogt, in: Kritik - Die Stimme des Volkes 75/92, S. 8.

95 Siehe in dieser Reihenfolge: Graf, in: Kritik - Die Stimme des Volkes 84/93, S. 63;

Honsik, in: Halt 60/91, S. 4; F. 61, S. 1, Z. 185 f. 
dieses Interesse, das dazu führt, dass Neologismen zur Diskreditierung kreiert werden: Sie dienen der Destruktion gegnerischer Wirklichkeit und der Konstruktion einer neuen Art und Weise des Sehens.

\section{Ausblick}

Wer seine Bezeichnung und Beschreibung der Realität, wer seine gedanklichen, seine argumentativen und sprachlichen Konstruktionen durchsetzt, der setzt damit auch seine Sichtweise durch - und schafft in diesem Sinne Wirklichkeit (Heringer 1990, S. 48). Um ihre Deutungen und Ideologeme zu kommunizieren und auf diese Weise Wirklichkeit zu schaffen, entwerfen neonazistische Gruppierungen, so ein genereller Befund dieser Analyse, eine eigene Gegensprache, die auf die Demontage missliebiger Auffassungen zielt. Versucht wird die Diskurskorrektur: Man möchte gegen die öffentliche Kommunikation über den Nationalsozialismus Front machen, will ein neues, ein besseres Image der eigenen Gruppe und Gesinnung aufbauen, um überhaupt die Basis für mögliche Persuasionserfolge und eine potentielle Mobilisierung von Sympathisanten $z \mathfrak{u}$ schaffen. Man möchte die öffentliche und die allgemeine Wahrnehmung zurechtrücken und initiiert regelrechte Wortkämpfe im Grenzbereich von Gruppen- und Gemeinsprache, um gegen Bezeichnungsvarianten vorzugehen, die aufgrund ihrer Wirkung und Verbreitung besonders irritieren - und eben nicht das ausdrücken, was wahrgenommen werden soll. Immer wieder fällt auf, in welchem Ausmaß Neonazis gegen die öffentliche Erinnerung an die Schrecken des Nationalsozialismus kämpfen. Sie versuchen, die Vergangenheit umzuschreiben, Geschichte anders zu deuten, Täter-Opfer-Verhältnisse neu zu konstruieren. Die Agenda ihrer Themen ist offensichtlich hochgradig extern bestimmt; man vermag sich nicht von der Deutungshoheit der Allgemeinheit abzukoppeln, die die eigenen Ziele in der großen Mehrheit ablehnt. Die minoritäre extremistische Subkultur wird durch die Mehrheitskultur nicht linear determiniert, aber doch, wenn man sich die beschriebenen Themen der Kommunikation erneut vergegenwärtigt, in den Inhalten der Auseinandersetzung regiert.

Die Kommunikation einer zahlenmäßig recht kleinen Gruppe von Extremisten, die sich hier offenbart, könnte man vermutlich auch am Beispiel anderer ideologischer, politischer und auch religiöser Vereinigungen, die öffentlich umstritten sind und gleichzeitig für ihre Sicht der Dinge werben wollen, beschreiben. Das wären dann - aus einer sprachund kommunikationswissenschaftlichen Perspektive - die Vorstudien zu einer Dramaturgie der politischen und ideologischen Auseinanderset- 
zung: Man würde jene Prozesse der Kommunikation dechiffrieren, die verhärtete Fronten, dogmatische Realitätsgewissheiten und letztlich vermutlich auch gewalttätige Ausbrüche entstehen lassen. Derartige Einsichten besitzen eine offenkundige Relevanz und sind nicht allein von wissenschaftlichem Interesse. Auch für die Existenz im Alltag und das Leben im Mikrokosmos des Privaten und im Makrokosmos des öffentlichen Raumes ist ihr Nutzen enorm.

\section{Quellen- und Literaturverzeichnis}

\subsection{Flugblätter und Flugschriften}

[Die Flugblätter und -schriften sind jeweils undatiert, aber nachweislich in den Jahren von 1989 bis 1993 erschienen; ihre Numerierung durch die neonazistische Gruppe Freundeskreis Freiheit für Deutschland wurde übernommen.]

Flugblatt 10: Warum schweigt man in Bonn?, S. 1-2.

Flugblatt 15: Höllenfeuer aus Menschenhand, S. 1-2.

Flugschrift 21: Antideutsche Ausrottungswaffen, S. 1-4.

Flugschrift 24: Jetzt schlägts dreizehn, S.1-3.

Flugblatt 25: Der verzweifelte Eiertanz der Auschwitz-Lügner, S. 1-2.

Flugblatt 26: In eigener Sache, S. 1-2.

Flugblatt 27: Deutsche aufgepaßt!, S. 1-2.

Flugblatt 31: Die Ausplïnderung des deutschen Volkes, S. 1-2.

Flugschrift 45: 200 (Zweihundert) Milliarden Deutsche Mark für eine Propagandalüge!?, S. 1-4.

Flugblatt 53: Widerstand!, S. 1-2.

Flugblatt 55: Kanzler Kohl voll auf Überfremdungskurs, S. 1-2.

Flugblatt 60: Volksverhetzung!, S. 1-2.

Flugblatt 61: Wir verlangen die Wahrheit und unser Recht - für unser Volk, S. 1-2.

Flugblatt 67: Wahlaufruf!, S. 1-2.

Flugblatt 95: Zwischen Mölln und Ahrensbök, S. 1-2.

Flugblatt 98: Bedeutendste historische Entdeckung des Jahres!, S. 1-2.

Flugblatt 99: Der Weg zur Wahrheit, S. 1-2.

Flugblatt 101: Bonn, wie es rülpst und lacht, S. 1-2.

Flugblatt 103: Staatsnotstand in Deutschland, S. 1-2.

Flugblatt 104: Die Wahrheit über das Asylunwesen, S. 1-2. 
5.2 Neonazistische Zeitungen und Zeitschriften

Aufbruch

11/91 Anonym: Fragen an die NF. S. 1-9.

Aufrecht! [Eigene Seitenzählung; Nummer und Erscheinungsjahr sind in den vorliegenden Ausgaben nicht lesbar]

Anonym: Leserbrief, S. 3.

Christian Wendt: FAP Kommentar, S. 12.

Deutsche Bürgerinitiative e. V. - weltweit [Eigene Seitennumerierung]

4/92 Manfred Roeder: Deutschlands Untergang ist beschlossen, S. 1-4.

Die Bauernschaft

2/89 Henning Fikentscher: [Brief Nummer] 17, S. 60.

4/90 Thies Christophersen: Liebe Freunde aus England, S. 23-26.

4/90 Max Drack: [Brief Nummer] 23, S. 55.

4/91 Hans Bielenberg: [Brief Nummer] 3. S. 51.

1/92 Germanicus: Der Fall Honsik vor dem OLG München, S. 12-13.

2/92 TAP und DRP: Mord, S. 3-11.

3/93 Arthur Vogt: Professor Faurisson, S. 11-13.

Eidgenoss

5/6/89 Anonym: Auschwitz und „Auschwitz-Lüge“, S. 5.

Halt [Eigene Seitennumerierung]

46/88 Gerd Honsik: Ich erhebe Einspruch, S. 1.

48/89 Anonym: Waterloo für Auschwitz, S. 4.

55/90 Anonym: Das Schandurteil, S. 1.

60/91 Gerd Honsik: Kanzler gegen Österreich, S. 4.

Kritik - Die Stimme des Volkes

75/92: Arthur Vogt: Der Holocaust - Legende oder Realität?, S. 7-30.

84/93 Jürgen Graf: Der Holocaust auf dem Prüfstand, S. 1-114.

Leitheft [Das Erscheinungsjahr der Zeitschrift ist nicht verzeichnet.

42 Ursula Bayrich: Keinen deutschen Pfennig für den amerikanischen Bombenmord an der irakischen Bevölkerung, S. 8-10.

Nachrichten der HNG

120/90 Erhard Kemper: [Unbetitelter Brief], S. 15-16.

143/92 Klaus Hellmund: Briefe an die Redaktion, S. 9-10.

149/93 Ursel Müller: Mitteilungen des Vorstandes, S. 6.

NS-Kampfruf

79/89 Germanicus: Betrachtungen über bundesrepublikanische Hilflosigkeiten am 20.4. 1989, S. 6. 


\section{Recht und Wahrheit}

3/4/90 G. A. Bosse: RuW-Interview, S. 12.

5/6/92 Ulrich Haberer: Umerziehung, S. 9-11.

Remer-Depesche

4/92 0. E. Remer: Liebe Landsleute, S. 4.

5/92 Fritz Stoß: Familie muß Asylanten aufnehmen, S. 1.

3/93 Anonym: Deutschenhasser und Fälscher in der Falle, S. 1.

3/93 Anonym: Juden wurden nicht vergast, S. 4.

\section{Sieg}

2/89 Walter Ochensberger: „Gaskammerprozeß“, S. 2-3.

2/89 Knud Deckert: Wille zur Macht!, S. 7-8.

4/5/89 Erich Kiwisch: Mein Aufruf an Deutschbewußte!, S. 21-22.

9/90 Anonym: Der Fünf-Fronten-Krieg, S. 9-10.

\section{Standarte}

4/93 Anonym: Der multikulturelle Selbstmord, S. 3-7.

\section{Tele-Blitz}

3/92 Anonym: Der Mai ist gekommen, und die politischen Ereignisse in Deutschland überschlagen sich, S. 1-4.

Unabhängige Nachrichten (UN)

2/89 Anonym: Die kalte Invasion, S. 6-8.

2/90 Siegfried Gärtner: Das Ausländerwahlrecht, S. 3-5.

9/90 W. Vogel: Man muß sich entscheiden, S. 4.

7/91 Berliner Jugendzeitung „Denkzettel“: Der Marsch in den „multikulturellen" Abgrund, S. 7

\section{Wehr' Dich!}

1/90 Anonym: Leser sagen ihre Meinung, S. 17.

5/92 Anonym: Nutznießer der Nation, S. 16.

\section{Wikinger}

1/89 Anonym: Natur und Glaube, S. 7-8.

3/89 Anonym: Europa - wie lange noch?, S. 29-30.

2/92 Anonym: Von Kamelen und Nadelöhren, S. 18-19.

2/92 Anonym: Ist das Morden von volkstreuen Deutschen ein Kavaliersdelikt?, S. 19-20.

2/92 Anonym: Neues aus Multikultopia. S. 20-21.

\subsection{Weitere Quellen}

Leuchter, Fred (1988): Leuchter-Report, Toronto: Samisdat. 


\subsection{Sekundärliteratur}

Bachem, Rolf (1979): Einführung in die Analyse politischer Texte, München: Oldenbourg

Bachem, Rolf (1983): Rechtsradikale Sprechmuster der 80er Jahre. Eine Studie zum Sprachgebrauch der "harten NS-Gruppen" und ihnen nahestehender Rechtsextremisten, in: Muttersprache, Jg. 93, S. $59-81$

Backes, Uwe (1998): Rechtsextremismus in Deutschland. Ideologien, Organisationen und Strategien, in: Aus Politik und Zeitgeschichte. Beilage zur Wochenzeitung ,Das Parlament', B 9-10, S. 27-35

Berning, Cornelia (1964): Vom „Abstammungsnachweis“ zum „Zuchtwart". Vokabular des Nationalsozialismus. Mit einem Vorwort von Werner Betz, Berlin: de Gruyter \& Co

Bott, Hermann (1969): Die Volksfeind-Ideologie. Zur Kritik rechtsradikaler Propaganda, Stuttgart: Deutsche Verlags-Anstalt (= Schriftenreihe der Vierteljahreshefte für Zeitgeschichte; 18)

Dieckmann, Walther (1964): Information oder Überredung. Zum Wortgebrauch der politischen Werbung in Deutschland seit der Französischen Revolution, Marburg: N. G. Elwert (= Marburger Beiträge zur Germanistik; 8)

Dietzsch, Martin (1988): Zwischen Konkurrenz und Kooperation. Organisationen und Presse der Rechten in der Bundesrepublik, in: Siegfried Jäger (Hg.): Rechtsdruck. Die Presse der Neuen Rechten, Berlin/ Bonn: Dietz, S. 31-80

Dudek, Peter/Jaschke, Hans-Gerd (1984): Entstehung und Entwicklung des Rechtsextremismus in der Bundesrepublik. Zur Tradition einer besonderen politischen Kultur. Bd 1, Westdeutscher Verlag: Opladen

Duve, Freimut (1994): Vom Krieg in der Seele. Rücksichten eines Deutschen, Frankfurt am Main: Eichborn

Foerster, Heinz von/Pörksen, Bernhard (1998): Wahrheit ist die Erfindung eines Lügners. Gespräche für Skeptiker, Heidelberg: Carl-AuerSysteme

Grünert, Horst (1974): Sprache und Politik. Untersuchungen zum Sprachgebrauch der ,Paulskirche', Berlin/New York: de Gruyter (= Studia Linguistica Germanica; 10)

Hannappel, Hans/Melenk, Hartmut (1990): Alltagssprache. Semantische Grundbegriffe und Analysebeispiele, Unveränderter Nachdruck der 2. überarbeiteten Aufl. von 1984, München: Fink

Hennig, Jörg (1994): Die Sprache der Medien und die politische Kultur in Deutschland. Vortrag beim ersten "dpa-Gespräch im Schloß" am 18. März 1994 [unveröffentlichtes Manuskript], S. 1-14 
Herberg, Dieter/Kinne Michael (1998): Neologismen, Heidelberg: Groos. (= Studienbibliographie Sprachwissenschaft; 23)

Heringer, Hans Jürgen (1990): „Ich gebe Ihnen mein Ehrenwort“. Politik, Sprache, Moral, München: Beck

Kaempfert, Manfred (1990): Die Schlagwörter. Noch einmal zur Wortgeschichte und zum lexikologischen Begriff, in: Muttersprache, Jg. 100, S. 192-203

Küster, Rainer (1983): Politische Metaphorik, in: Sprache und Literatur in Wissenschaft und Unterricht (vormals "Linguistik und Didaktik“), Jg. 14, H. 51, S. 30-45

Küster, Rainer (1989): Mythische Aspekte militärischer Metaphorik, in: Armin Burkhardt/Franz Hebel/Rudolf Hoberg: Sprache zwischen Militär und Frieden: Aufrüstung der Begriffe?, Tübingen: Narr (= Forum für Fachsprachenforschung; 7), S. 81-92

Lübbe, Hermann (1975): Streit um Worte, in: Gerd-Klaus Kaltenbrunner (Hg.): Sprache und Herrschaft. Die umfunktionierten Wörter, München: Herder (= Herderbücherei INITIATIVE; 5), S. 87-111

Mayr, Monika (1993): Dresden, in: Wolfgang Benz (Hg.): Legenden, Lügen, Vorurteile. Ein Wörterbuch zur Zeitgeschichte, 3. Aufl. München: Deutscher Taschenbuch Verlag, S. 61-62

Piper, Ernst (1995): Achtes Bild: „Die jüdische Weltverschwörung“, in: Julius H. Schoeps/Joachim Schlör (Hg.): Antisemitismus. Vorurteile und Mythen, München/Zürich: Piper, S. 127-135

Pörksen, Bernhard (1997): „Wir können von der Wirklichkeit nur wissen, was sie nicht ist." Im Gespräch mit Paul Watzlawick, in: Communicatio Socialis, Jg. 30, S. 383-399

Pörksen, Bernhard (2000): Die Konstruktion von Feindbildern. Zum Sprachgebrauch in neonazistischen Medien, Wiesbaden: Westdeutscher Verlag

Pörksen, Bernhard (2001): Gegensprache, in: Dieter, Möhn/Dieter Roß/ Marita Tjarks-Sobhani: Mediensprache und Medienlinguistik. Festschrift für Jörg Hennig, Frankfurt am Main u.a.: Peter Lang, S. 311-337

Pörksen, Uwe (1974): Textsorte, Textsortenverschränkungen und Sprachattrappen, in: Wirkendes Wort, Jg. 24, S. 219-239

Römer, Ruth (1990): Sprachkritische Anmerkungen zum Historikerstreit, in: Muttersprache, Jg. 100, S. 259-265

Schmidt, Michael (1993): Heute gehört uns die Straße ... Der InsideReport aus der Neonazi-Szene, Düsseldorf - Wien - New York - Moskau: Econ

Stötzel, Georg (1995): Der Nazi-Komplex, in: Georg Stötzel/Martin Wengeler (Hg.): Kontroverse Begriffe. Geschichte des öffentlichen Sprach- 
gebrauchs in der Bundesrepublik Deutschland, Berlin - New York: de Gruyter (= Sprache, Politik, Öffentlichkeit; 4), S. 355-382

Virchow, Fabian (1996): „... über die Trümmer der KZ-Gedenkstätten“. Von Auschwitzleugnern und anderen Geschichtsfälschern, in: Jens Mecklenburg (Hg.): Handbuch deutscher Rechtsextremismus, Berlin: Elefanten Press, S. 666-691 